DCPT-06/41

hep-th/0612298

\title{
Pseudo-differential equations, and the Bethe Ansatz for the classical Lie algebras
}

\author{
Patrick Dorey ${ }^{1}$, Clare Dunning $^{2}$, Davide Masoero $^{3}$, Junji Suzuki $^{4}$ and Roberto Tateo ${ }^{5}$ \\ ${ }^{1}$ Dept. of Mathematical Sciences, University of Durham, \\ Durham DH1 3LE, United Kingdom \\ ${ }^{2}$ IMSAS, University of Kent, Canterbury, UK CT2 7NF, United Kingdom \\ ${ }^{3}$ SISSA, via Beirut 2-4, 34014 Trieste, Italy \\ ${ }^{4}$ Department of Physics, Shizuoka University, Ohya 836, SURUGA, Shizuoka, Japan. \\ ${ }^{5}$ Dip. di Fisica Teorica and INFN, Università di Torino, \\ Via P. Giuria 1, 10125 Torino, Italy \\ E-mails: \\ p.e.dorey@durham.ac.uk, t.c.dunning@kent.ac.uk, \\ masoero@sissa.it, sjsuzuk@ipc.shizuoka.ac.jp, tateo@to.infn.it
}

\begin{abstract}
The correspondence between ordinary differential equations and Bethe ansatz equations for integrable lattice models in their continuum limits is generalised to vertex models related to classical simple Lie algebras. New families of pseudo-differential equations are proposed, and a link between specific generalised eigenvalue problems for these equations and the Bethe ansatz is deduced. The pseudo-differential operators resemble in form the Miuratransformed Lax operators studied in work on generalised KdV equations, classical $\mathrm{W}$-algebras and, more recently, in the context of the geometric Langlands correspondence. Negative-dimension and boundary-condition dualities are also observed.
\end{abstract}

PACS: 03.65.-Ge, 11.15.Tk, 11.25.HF, 11.55.DS.

Keywords: conformal field theory, Bethe ansatz, pseudo-differential equations, spectral problems. 


\section{Introduction}

A recent observation [1] has established an unexpected link between two dimensional conformal field theory (CFT) and the theory of ordinary differential equations. This rests on a correspondence between the transfer matrix eigenvalues of certain integrable models (IMs), in their conformal limits [2, 3], and the spectral determinants [4, 5] of ordinary differential equations.

The initial results [1, 6, 7, 8, connected conformal field theories with Virasoro central charge $c \leq 1$ with Schrödinger problems for one-dimensional anharmonic oscillators. These conformal field theories are naturally associated to the Lie algebra $A_{1}$, but a generalisation to models related to $A_{n-1}$, with additional extended $\mathrm{W}$-algebra symmetries, was soon established [9, 10, 11, 12. However, contrary to initial expectations, a simple Lie-algebraic structure did not emerge immediately, and the extension of the correspondence to the theories associated with other simple Lie algebras $\mathfrak{g}$ has proved surprisingly elusive.

The purpose of this paper is to begin to fill this gap, by establishing a link between CFTs related to the classical simple Lie algebras and spectral problems associated with a set of ordinary (pseudo-) differential equations.

We shall also prove for $\mathfrak{g}=A_{n-1}$, and conjecture for the other simple Lie algebras, the existence of closed systems of functional equations ( $\psi$-systems) among uniquely-defined solutions $\psi^{(1)}, \psi^{(2)}, \ldots, \psi^{(\operatorname{rank}(\mathfrak{g}))}$ of a set of $\operatorname{rank}(\mathfrak{g})$ pseudodifferential operators, with each pair $\psi^{(a)} /$ (operator) $)_{a}$ being naturally associated to a node of the Dynkin diagram. These $\psi$-systems are very similar to the systems of functional relations introduced by Mukhin and Varchenko in the framework of the Bethe ansatz (BA) method for $\mathfrak{g}-\mathrm{XXX}$ quantum spin chains [13, 14, 15, 16], and in the context of the so-called Miura-opers related to the geometric Langlands correspondence (see, for example, [17, 18]). This similarity is related to the fact that the homogeneous 'differential' parts of the operators studied here resemble, in form, the Miura-transformed Lax operators introduced by Drinfel'd and Sokolov in their studies of generalised $\mathrm{KdV}$ equations and classical W-algebras [19].

The rest of the paper is organised as follows. \$2 gathers together some known, or easily deduced, properties of the Bethe equations for $\mathfrak{g}$-type quantum spin chains in their continuum limits. Our main results are summarised in 93 , while extra details and numerical support for the specific $A, D, B$ and $C$ proposals are given in $\$ 4$ to $\$ 7$ respectively, and $\$ 8$ contains our general conclusions. There are two appendices: appendix $\mathrm{A}$ deals with the semiclassical analysis for $A_{1}$-related ODEs in the presence of string solutions, and appendix $B$ describes a simple algorithm useful for the numerical solutions of the differential equations. The algorithm is a generalisation of Cheng's method from Regge pole theory [20], and relies on an elegant dual formulation of the relevant boundary problems. 


\section{The Bethe Ansatz equations and their string solu- tions}

For any simple Lie algebra $\mathfrak{g}$ of type $A_{n-1}$ to $G_{2}$, a set of Bethe ansatz equations (BAEs), depending on a set of $\operatorname{rank}(\mathfrak{g})$ twist parameters $\gamma=\left\{\gamma_{a}\right\}$, can be written in a universal form as $[21,22,23,24,25,26]^{*}$

$$
\prod_{b=1}^{\operatorname{rank}(\mathfrak{g})} \Omega^{B_{a b} \gamma_{b}} \frac{Q_{B_{a b}}^{(b)}\left(E_{i}^{(a)}, \gamma\right)}{Q_{-B_{a b}}^{(b)}\left(E_{i}^{(a)}, \gamma\right)}=-1, \quad i=0,1,2, \ldots
$$

where

$$
Q_{k}^{(a)}(E, \gamma)=Q^{(a)}\left(\Omega^{k} E, \gamma\right)
$$

and the numbers $E_{i}^{(a)}$ are the - in general complex - zeros of the functions $Q^{(a)}$ :

$$
Q^{(a)}\left(E_{i}^{(a)}, \gamma\right)=0
$$

In (2.1) and (2.3) the indices $a$ and $b$ label the simple roots of the Lie algebra, the matrix $B_{a b}$ is defined by

$$
B_{a b}=\frac{\left(\alpha_{a}, \alpha_{b}\right)}{\mid \operatorname{long} \text { roots }\left.\right|^{2}}, \quad a, b=1,2, \ldots, \operatorname{rank}(\mathfrak{g})
$$

and the $\alpha$ 's are the simple roots of $\mathfrak{g}$. The constant $\Omega$ is a pure phase, parameterised in terms of a real number $\mu>0$ as

$$
\Omega=\exp \left(i \frac{2 \pi}{h^{\vee} \mu}\right)
$$

where $h^{\vee}$ is the dual Coxeter number of $\mathfrak{g}$. Strictly speaking, the BAE (2.1) arise from taking a suitable continuum or field theory limit of the lattice model BAE, in the fashion explained in, for example, [27].

The functions $Q^{(a)}$ appearing in (2.1) have a characteristic asymptotic behaviour at large values of $-E$

$$
\ln Q^{(a)}(-E, \gamma)=m_{a} \frac{\sin \left(\frac{\pi}{h^{\vee}}\right)}{\sin \left(\frac{\pi}{h^{\nabla}} B_{a a}\right)}(-E)^{\mu}+\ldots .
$$

For the $A_{n-1}, B_{n}, C_{n}$ and $D_{n}$ models the sets $\left\{m_{a}\right\}$ are given in table 1 丹 The only free parameter -the overall constant $m$ in table 1- depends on the way the conformal field theory limit is reached.

${ }^{*}$ For finite lattice models, the explicit diagonalisation of the $A_{n-1}$ cases has been performed through the algebraic Bethe ansatz by Schulz 21 and also by Babelon, de Vega and Viallet 22. For $C_{n}$ and $D_{n}$ models, it has been done by Reshetikhin 23, 24]. There is a shortcut to reach the same conclusions via the so-called analytic Bethe ansatz of Reshetikhin [25], and Wiegmann and Reshetikhin [26].

$\dagger$ The constants $\left\{m_{a}\right\}$ are related to a particular matrix $K_{a b}$ emerging from the analysis of the Bethe ansatz. For simply-laced algebras, $K_{a b}$ is proportional to the Cartan matrix and $\vec{v}=\left(m_{1}, m_{2}, \ldots, m_{r}\right)$ is its Perron-Frobenius eigenvector. 


\begin{tabular}{|c|c|c|}
\hline Model & $h^{\vee}$ & $m_{a}$ \\
\hline \hline$A_{n-1}$ & $n$ & $m_{a}=2 m \sin \left(a \frac{\pi}{h^{\vee}}\right),(a=1, \ldots, n-1)$ \\
\hline$D_{n}$ & $2 n-2$ & $m_{n-1}=m_{n}=m, m_{a}=2 m \sin \left(a \frac{\pi}{h^{\vee}}\right),(a=1, \ldots, n-2)$ \\
\hline$B_{n}$ & $2 n-1$ & $m_{n}=m, m_{a}=2 m \sin \left(a \frac{\pi}{h^{\vee}}\right),(a=1, \ldots, n-1)$ \\
\hline$C_{n}$ & $n+1$ & $m_{a}=2 m \sin \left(a \frac{\pi}{2 h^{\vee}}\right),(a=1, \ldots, n)$ \\
\hline
\end{tabular}

Table 1: Dual Coxeter numbers and coefficients $\left\{m_{a}\right\}$ for models based on classical simple Lie algebras.

The negative real $E$ axis is also the direction of maximal growth for $\ln Q^{(a)}(E)$ as $|E| \rightarrow \infty$. From (2.6), the Hadamard order of $Q^{(a)}$ is therefore $\mu$ and, in the so-called 'semiclassical regime' $0<\mu<1, Q^{(a)}$ can be written as a convergent infinite product over its zeros as

$$
Q^{(a)}(E, \gamma)=Q^{(a)}(0, \gamma) \prod_{i=0}^{\infty}\left(1-\frac{E}{E_{i}^{(a)}}\right)
$$

It turns out that the Bethe ansatz roots generally split into multiplets with approximately equal modulus $\left|E_{i}^{(a)}\right|$, and that the ground state of the quantum spin chain corresponds to a 'pure' configuration of roots, containing only multiplets with a common dimension

$$
d_{a}=\frac{K}{B_{a a}} .
$$

The integer $K$ in (2.8) depends on the particular spin chain under discussion, and corresponds to the degree of fusion [28, 29, 30]. For $\mathfrak{g}=A_{1}$, the spin- $j A_{1}$ quantum chains, $K=d_{1}=2 j$.

It is generally expected [31] that for large values of $i$ the zeros asymptotically tend to the perfect string configurations:

$$
\arg E_{i}^{(a)} \sim\left(d_{a}+1-2 l\right) \frac{B_{a a} \pi}{h^{\vee} \mu}, \quad l=1,2, \ldots, d_{a} .
$$

Appendix A contains some further discussion of the asymptotic behaviour of these string solutions. 


\section{Summary of the main results}

This paper is about the correspondence between the Bethe ansatz equations (2.1) for $\mathfrak{g}=A_{n-1}, B_{n}, C_{n}, D_{n}$ and spectral problems associated to solutions $\psi(x, E, \mathbf{g})$ of particular pseudo-differential equations with vanishing boundary conditions

$$
\psi(x, E, \mathbf{g})=o\left(e^{-\frac{x^{M+1}}{M+1}}\right) \quad, \quad\left(M>K /\left(h^{\vee}-K\right)\right)
$$

imposed at large $x$ on the positive real axis. To specify these equations we introduce the $n^{\text {th }}$-order differential operator [1]

$$
\begin{gathered}
D_{n}(\mathbf{g})=D\left(g_{n-1}-(n-1)\right) D\left(g_{n-2}-(n-2)\right) \ldots D\left(g_{1}-1\right) D\left(g_{0}\right), \\
D(g)=\left(\frac{d}{d x}-\frac{g}{x}\right),
\end{gathered}
$$

with

$$
\mathbf{g}=\left\{g_{n-1}, \ldots, g_{1}, g_{0}\right\} \quad, \mathbf{g}^{\dagger}=\left\{n-1-g_{0}, n-1-g_{1}, \ldots, n-1-g_{n-1}\right\} .
$$

We also use the inverse differential operator $(d / d x)^{-1}$, generally defined through its formal action

$$
\left(\frac{d}{d x}\right)^{-1} x^{s}=\frac{x^{s+1}}{s+1}
$$

The following properties hold

$$
\left(\frac{d}{d x}\right)\left(\frac{d}{d x}\right)^{-1} x^{s}=x^{s}, \quad\left(\frac{d}{d x}\right)^{-1}\left(\frac{d}{d x}\right) x^{s}=x^{s} \quad(s \neq 0),
$$

and the integration by parts property

$$
\left(\frac{d}{d x}\right)^{-1}\left[f(x) \frac{d}{d x} g(x)\right]=f(x) g(x)-\left(\frac{d}{d x}\right)^{-1}\left[g(x) \frac{d}{d x} f(x)\right]
$$

is satisfied, provided in the $x$-expansion of $f(x) g(x)$ about the origin the constant term is absent. In the following we shall assume the validity of (3.16) by working implicitly with non-integer values for the parameters $g_{i}$ introduced in (3.13), and by invoking continuity of the final results in these parameters.

Finally, we define a basic 'potential'

$$
P_{K}(E, x)=\left(x^{h^{\vee} M / K}-E\right)^{K} .
$$


With this notation in place, the following pseudo-differential equations are the main concern of this article:

$$
A_{n-1}(\mathbf{s u}(\mathbf{n}))
$$

$$
\left((-1)^{n} D_{n}(\mathbf{g})-P_{K}(x, E)\right) \psi(x, E, \mathbf{g})=0,
$$

with the constraint $\sum_{i=0}^{n-1} g_{i}=\frac{n(n-1)}{2}$.

$$
\begin{aligned}
& D_{n}(\mathbf{s o}(\mathbf{2 n})): \\
& \left(D_{n}\left(\mathbf{g}^{\dagger}\right)\left(\frac{d}{d x}\right)^{-1} D_{n}(\mathbf{g})-\sqrt{P_{K}(x, E)}\left(\frac{d}{d x}\right) \sqrt{P_{K}(x, E)}\right) \psi(x, E, \mathbf{g})=0
\end{aligned}
$$

$$
\begin{aligned}
& B_{n}(\mathbf{s o}(\mathbf{2 n}+\mathbf{1})): \\
& \quad\left(D_{n}\left(\mathbf{g}^{\dagger}\right) D_{n}(\mathbf{g})+\sqrt{P_{K}(x, E)}\left(\frac{d}{d x}\right) \sqrt{P_{K}(x, E)}\right) \psi(x, E, \mathbf{g})=0
\end{aligned}
$$

\section{$C_{n}(\operatorname{sp}(2 \mathbf{n})):$}

$$
\left(D_{n}\left(\mathbf{g}^{\dagger}\right)\left(\frac{d}{d x}\right) D_{n}(\mathbf{g})-P_{K}(x, E)\left(\frac{d}{d x}\right)^{-1} P_{K}(x, E)\right) \psi(x, E, \mathbf{g})=0
$$

The correspondence we propose links the ground-state $Q^{(1)}$ 's of (2.1) to particular solutions $\psi(3.10)$ of equations (3.18 3.21).

In order to clarify this statement we introduce an alternative basis of solutions $\left\{\chi_{i}(x, E, \mathbf{g})\right\}$ to (3.18 3.21), characterised by their behaviour near the origin

$$
\chi_{i}(x, E, \mathbf{g}) \sim x^{\lambda_{i}}+\ldots \quad, \quad x \rightarrow 0,
$$

where the $\lambda$ 's are the ordered $\left(\lambda_{0}<\lambda_{1}<\ldots\right)$ roots of the appropriate indicial equation (see table 2).

Writing $\psi$ as a linear combination of the $\chi$ 's, we have in general

$$
\psi(x, E, \mathbf{g})=Q_{[0]}^{(1)}(E, \mathbf{g}) \chi_{0}(x, E, \mathbf{g})+Q_{[1]}^{(1)}(E, \mathbf{g}) \chi_{1}(x, E, \mathbf{g})+\ldots .
$$

If the zeros of $Q_{[0]}^{(1)}(E, \mathbf{g})$ are $\left\{E_{i}^{(1)}\right\}$, then for $E \in\left\{E_{i}^{(1)}\right\}$ the function $x^{-\lambda_{0}} \psi(x, E, \mathbf{g})$ vanishes exceptionally both at $x=\infty$ and at $x=0$. This allows the coefficient 


\begin{tabular}{|c|c|}
\hline Model & indicial equation \\
\hline \hline$A_{n-1}$ & $\prod_{i=0}^{n-1}\left(\lambda-g_{i}\right)=0$ \\
\hline$D_{n}$ & $\left(\lambda-h^{\vee} / 2\right)^{-1} \prod_{i=0}^{n-1}\left(\lambda-g_{i}\right)\left(\lambda-h^{\vee}+g_{i}\right)=0$ \\
\hline$B_{n}$ & $\prod_{i=0}^{n-1}\left(\lambda-g_{i}\right)\left(\lambda-h^{\vee}+g_{i}\right)=0$ \\
\hline$C_{n}$ & $(\lambda-n) \prod_{i=0}^{n-1}\left(\lambda-g_{i}\right)\left(\lambda-2 n+g_{i}\right)=0$ \\
\hline
\end{tabular}

Table 2: Indicial equations.

$Q_{[0]}^{(1)}(E, \mathbf{g})$ to be identified with the spectral determinant for a boundary problem defined on the positive real axis (see, for example, [4, 5]). An alternative (dual) definition of the spectral functions $Q_{[0]}^{(1)}(E, \mathbf{g})$ in terms of the adjoint equations to (3.18)-(3.21) is briefly discussed in appendix B.

We claim that for classical Lie algebras with arbitrary degree of fusion $K$, the ground-state $Q^{(1)}(E, \gamma)$ 's in (2.1) and the functions $Q_{[0]}^{(1)}(E, \mathbf{g})$ in (3.23) coincide up to a trivial normalisation, so that

$$
\frac{Q^{(1)}(E, \gamma)}{Q^{(1)}(0, \gamma)}=\frac{Q_{[0]}^{(1)}(E, \mathbf{g})}{Q_{[0]}^{(1)}(0, \mathbf{g})}
$$

Moreover, from the WKB approximation

$$
\ln Q_{[0]}^{(1)}(-E, \mathbf{g})=\kappa(-E)^{\widehat{\mu}}+\ldots \quad(E \gg 0)
$$

with

$$
\widehat{\mu}=\frac{K(M+1)}{h^{\vee} M}, \quad \kappa=\kappa\left(\frac{h^{\vee} M}{K}, \frac{h^{\vee}}{K}\right)
$$

and

$$
\kappa(a, b)=\int_{0}^{\infty} d x\left(\left(x^{a}+1\right)^{b}-x^{a b}\right)=\frac{\Gamma(1+1 / a) \Gamma(1+1 / b) \sin (\pi / b)}{\Gamma(1+1 / a+1 / b) \sin (\pi / b+\pi / a)} .
$$

Therefore, in order to have a match between (3.25) and (2.6), we must set

$$
\mu=\widehat{\mu}, \quad m_{1}=\kappa \frac{\sin \left(\frac{\pi}{h^{\nabla}} B_{11}\right)}{\sin \left(\frac{\pi}{h^{\vee}}\right)}, \quad \Omega=\exp \left(i \frac{2 \pi M}{K(M+1)}\right) .
$$

Given a particular ordering convention, the relationship between the twist parameters $\left\{\gamma_{a}\right\}$ and the constants $\left\{g_{a}\right\}$ is given in table 3 , 


\begin{tabular}{|c|c|c|}
\hline Model & ordering $\forall i<j$ & $\left\{g_{a}\right\} \leftrightarrow\left\{\gamma_{a}\right\}$ \\
\hline \hline$A_{n-1}$ & $g_{i}<g_{j}$ & $\gamma_{a}=\alpha\left(\sum_{i=0}^{a-1} g_{i}-\frac{a\left(h^{\vee}-1\right)}{2}\right)$ \\
\hline & & $\gamma_{a}=\alpha\left(\sum_{i=0}^{a-1} g_{i}-\frac{a}{2} h^{\vee}\right),(a=1, \ldots, n-2)$ \\
$D_{n}$ & $g_{i}<g_{j}<h^{\vee} / 2$ & $\gamma_{n-1}=\frac{\alpha}{2}\left(\sum_{i=0}^{n-1} g_{i}-\frac{n}{2} h^{\vee}\right)$ \\
& & $\gamma_{n}=\frac{\alpha}{2}\left(\sum_{i=0}^{n-2} g_{i}-g_{n-1}-\frac{n-2}{2} h^{\vee}\right)$ \\
\hline$B_{n}$ & $g_{i}<g_{j}<h^{\vee} / 2$ & $\gamma_{a}=\alpha\left(\sum_{i=0}^{a-1} g_{i}-\frac{a}{2} h^{\vee}\right)$ \\
\hline$C_{n}$ & $g_{i}<g_{j}<n$ & $\gamma_{a}=\alpha\left(\sum_{i=0}^{a-1} g_{i}-a n\right)$ \\
& & $\gamma_{n}=\frac{\alpha}{2}\left(\sum_{i=0}^{n-1} g_{i}-n^{2}\right)$ \\
\hline
\end{tabular}

Table 3: The relationship between the set of parameters $\left\{g_{a}\right\} \leftrightarrow\left\{\gamma_{a}\right\}$ with $\alpha=2 K / M h^{\vee}$.

Various consistency checks, including the WKB approach and numerical work, support the correspondence both qualitatively and quantitatively.

Finally, starting from equations (3.18) to (3.21), the Bethe ansatz equations and table 3 were obtained with the help of a system of functional relations involving $\psi^{(1)}(x, E, \mathbf{g})=\psi(x, E, \mathbf{g})$ together with other auxiliary functions $\psi^{(a)}(x, E, \mathbf{g}),(a=2, \ldots, \operatorname{rank}(\mathfrak{g}))$ (see $₫ 4-87$ and [9, 11]). We setf

$$
\psi_{k}^{(a)}=\psi^{(a)}\left(\omega^{k} x, \Omega^{k} E, \mathbf{g}\right)
$$

where

$$
\Omega=\exp \left(i \frac{2 \pi M}{K(M+1)}\right)
$$

as in (3.28), and

$$
\omega=\Omega^{K / h^{\vee}}=\exp \left(i \frac{2 \pi}{h^{\vee}(M+1)}\right) .
$$

For the simply-laced algebras the $\psi$-systems can then be written in the compact form

$$
W\left[\psi_{-\frac{1}{2}}^{(a)}, \psi_{\frac{1}{2}}^{(a)}\right]=\prod_{b=1}^{\operatorname{rank}(\mathfrak{g})}\left(\psi^{(b)}\right)^{A_{a b}},
$$

\footnotetext{
${ }^{\ddagger}$ In the $A_{n-1}$ models $\psi_{k}^{(1)}(x, E, \mathbf{g})=\omega^{(n-1) k / 2} y_{-k}(x, E, \mathbf{g})$, where $y_{k}$ is the function defined in $\S 3$ of 11 .
} 
where $A_{a b}=2 \delta_{a b}-2 B_{a b}$ is the incidence matrix of the corresponding Dynkin diagram and $W$ the Wronskian:

$$
W[f, g]=f(x) \frac{d}{d x} g(x)-g(x) \frac{d}{d x} f(x) .
$$

Equation (3.32) is proven in $\$ 4.2$ for $\mathfrak{g}=A_{n-1}$, and our numerical results indirectly support the validity of (3.32) for $\mathfrak{g}=D_{n}$. Currently, we have no analogous pseudodifferential equations for the exceptional Lie algebras but the similarity between (3.32), the relations proposed in [13, 14, 15] and the other functional equations (Y-systems and T-systems) discovered in the framework of integrable models [32, 33, 34, 35, 36, 37] suggests the validity of (3.32) in its more general form. For the non simply-laced algebras our conjectures are

$$
\begin{aligned}
B_{n}: \quad W\left[\psi_{-\frac{1}{2}}^{(a)}, \psi_{\frac{1}{2}}^{(a)}\right] & =\psi^{(a-1)} \psi^{(a+1)} ; \quad a=1, \ldots, n-1, \\
W\left[\psi_{-\frac{1}{4}}^{(n)}, \psi_{\frac{1}{4}}^{(n)}\right] & =\psi_{-\frac{1}{4}}^{(n-1)} \psi_{\frac{1}{4}}^{(n-1)} .
\end{aligned}
$$

(Our root convention is $\left(\alpha_{i} \mid \alpha_{i}\right)=2$ for $i=1,2, \ldots, n-1$ and $\left(\alpha_{n} \mid \alpha_{n}\right)=1$.)

$$
\begin{aligned}
C_{n}: \quad & W\left[\psi_{-\frac{1}{4}}^{(a)}, \psi_{\frac{1}{4}}^{(a)}\right]=\psi^{(a-1)} \psi^{(a+1)} ; \quad a=1, \ldots, n-2, \\
& W\left[\psi_{-\frac{1}{4}}^{(n-1)}, \psi_{\frac{1}{4}}^{(n-1)}\right]=\psi^{(n-2)} \psi_{-\frac{1}{4}}^{(n)} \psi_{\frac{1}{4}}^{(n)}, \\
& W\left[\psi_{-\frac{1}{2}}^{(n)}, \psi_{\frac{1}{2}}^{(n)}\right]=\psi^{(n-1)} .
\end{aligned}
$$

(Here, $\left(\alpha_{i} \mid \alpha_{i}\right)=1$ for $i=1,2, \ldots, n-1$ and $\left(\alpha_{n} \mid \alpha_{n}\right)=2$.)

$$
\begin{aligned}
& F_{4}: \quad W\left[\psi_{-\frac{1}{4}}^{(1)}, \psi_{\frac{1}{4}}^{(1)}\right]=\psi^{(2)}, \\
& W\left[\psi_{-\frac{1}{4}}^{(2)}, \psi_{\frac{1}{4}}^{(2)}\right]=\psi^{(1)} \psi_{-\frac{1}{4}}^{(3)} \psi_{\frac{1}{4}}^{(3)}, \\
& W\left[\psi_{-\frac{1}{2}}^{(3)}, \psi_{\frac{1}{2}}^{(3)}\right]=\psi^{(2)} \psi^{(4)} \\
& W\left[\psi_{-\frac{1}{2}}^{(4)}, \psi_{\frac{1}{2}}^{(4)}\right]=\psi^{(3)} .
\end{aligned}
$$

(Here, $\left(\alpha_{1}, \alpha_{1}\right)=\left(\alpha_{2}, \alpha_{2}\right)=1$ and $\left(\alpha_{3}, \alpha_{3}\right)=\left(\alpha_{4}, \alpha_{4}\right)=2$.)

$$
\begin{aligned}
G_{2}: & W\left[\psi_{-\frac{1}{2}}^{(1)}, \psi_{\frac{1}{2}}^{(1)}\right]=\psi^{(2)}, \\
& W\left[\psi_{-\frac{1}{6}}^{(2)}, \psi_{\frac{1}{6}}^{(2)}\right]=\psi^{(1)} \psi_{-\frac{2}{6}}^{(1)} \psi_{\frac{2}{6}}^{(1)} .
\end{aligned}
$$

(Here, $\left(\alpha_{1} \mid \alpha_{1}\right)=3$ and $\left(\alpha_{2} \mid \alpha_{2}\right)=1$.) 
Again, these relations are not proven but we have indirect numerical evidence for $\mathfrak{g}=B_{n}, C_{n}$. Further details and numerical support for the above conjectures are provided in the following sections, which examine the $A, D, B$ and $C$ cases in turn.

\section{The $A_{n-1}$ models}

The ODE for the $A_{n-1}$ models is

$$
\left((-1)^{n+1} D_{n}(\mathbf{g})+P_{K}(x, E)\right) \psi(x, E, \mathbf{g})=0,
$$

where the operator $D_{n}(\mathbf{g})$ and the generalised potential $P_{K}(x, E)$ were defined in \$3, and the additional constraint

$$
\sum_{i=0}^{n-1} g_{i}=\frac{n(n-1)}{2}
$$

ensures that the term $x^{-1} \frac{d^{n-1}}{d x^{n-1}}$ is absent.

The function $\psi(x, E, \mathbf{g})$ is defined to be the most subdominant solution on the positive real axis, with asymptotic behaviour, for $M>K /\left(h^{\vee}-K\right)$, given by

$$
\psi(x, E, \mathbf{g}) \sim \mathcal{N} x^{(1-n) M / 2} \exp \left(-x^{M+1} /(M+1)\right)
$$

as $x \rightarrow \infty$. The coefficient $\mathcal{N}$ represents an $E$ - and $\mathbf{g}$-independent normalisation constant.

The $K=1$ cases have been extensively discussed in [9, 10, 11, 12]; they are related to the $W A_{n-1}$ conformal field theories with integer Virasoro central charge $c=n-1$. Alternatively, at particular values of the parameters $\mathbf{g}$ and $M$ they can also be put in correspondence with the minimal coset conformal field theories

$$
\frac{\left(\widehat{A}_{n-1}\right)_{1} \times\left(\widehat{A}_{n-1}\right)_{L}}{\left(\widehat{A}_{n-1}\right)_{L+1}},
$$

with a simple relationship between $L$ in (4.4) and the parameter $M$ in (3.17).

The generalisation to integer $K>1$ comes from an observation by Sergei Lukyanov [38. for the $A_{1}$ case, for which numerical and analytic support was later provided in [39] and in [40]. It is reasonable to conjecture that this generalisation works both for $A_{n-1}$ with $n>2$ and, up to minor modifications, for the other models to be discussed in this paper. In analogy to (4.4), at particular values of $\mathbf{g}$ and $M$, the integer $K>1$ cases should correspond to the cosets

$$
\frac{\widehat{\mathfrak{g}}_{K} \times \widehat{\mathfrak{g}}_{L}}{\widehat{\mathfrak{g}}_{K+L}},
$$

which describe conformal field theories with $\mathfrak{g}=A_{n-1}, B_{n}, C_{n}, D_{n}$. In appendix $\mathrm{A}$ we shall explain, in the simplest case, why potentials of such forms naturally lead to string patterns of roots of the sort mentioned in the introduction. 


\subsection{Negative-dimension dualities}

It is interesting to note that there are formal duality relations among our pseudodifferential equations involving negative values of $n$ and $K$. Consider the $A_{n-1}$ ODEs with the twists $\mathbf{g}=\{0,1, \ldots, n-1\}$ :

$$
\left((-1)^{n+1} \frac{d^{n}}{d x^{n}}+P_{K}(x, E)\right) \psi(x, E)=0 .
$$

Setting $\tilde{\psi}(x, E)=P_{K}(x, E) \psi(x, E)$ and multiplying from the left by $\left(\frac{d}{d x}\right)^{-n}$, the result is

$$
\left((-1)^{n+1}\left(\frac{d}{d x}\right)^{-n}+P_{-K}(x, E)\right) \tilde{\psi}(x, E)=0 .
$$

Comparing (4.7) with (4.6) and taking into account the boundary conditions, we see that there is a formal duality and a spectral equivalence between the initial $n^{\text {th }}$-order ODE and the pseudo-differential equation (4.7):

$$
\{n, M, K\} \leftrightarrow\{-n, M,-K\}
$$

Though the above manipulation might look purely formal, it strongly resembles previously-observed W-algebra dualities [41]:

$$
\frac{\left(\widehat{A}_{-n}\right)_{K} \times\left(\widehat{A}_{-n}\right)_{L}}{\left(\widehat{A}_{-n}\right)_{K+L}} \sim \frac{\left(\widehat{A}_{n}\right)_{-K} \times\left(\widehat{A}_{n}\right)_{-L}}{\left(\widehat{A}_{n}\right)_{-K-L}} .
$$

A discussion of the precise relation between $L$ and the ODE parameters $\{n, M, K, \mathbf{g}\}$ is not important for the current naive considerations and we shall postpone it to the future.

Whilst the duality (4.8) remains at the moment a purely formal observation, the second duality discussed in [41]

$$
\frac{\left(\widehat{D}_{-n}\right)_{K} \times\left(\widehat{D}_{-n}\right)_{L}}{\left(\widehat{D}_{-n}\right)_{K+L}} \sim \frac{\left(\widehat{C}_{n}\right)_{-K / 2} \times\left(\widehat{C}_{n}\right)_{-L / 2}}{\left(\widehat{C}_{n}\right)_{-K / 2-L / 2}},
$$

will lead us to the proposal (3.21) for the $C_{n}$-related equations. (For the quantisation of the classical $D_{n} \mathrm{~W}$-algebras in relation to the Miura-opers see, for example, 42].)

Negative-dimension dualities resembling those described here are also wellknown to group theorists; many more details can be found in [43].

\subsection{Auxiliary functions and the $\psi$-system}

The solution $\psi(x, E, \mathbf{g})$ of (4.1) with the asymptotic behavior (4.3) is not the only function associated to the $A_{n-1}$ Bethe ansatz equations. In order to derive the Bethe ansatz itself a total of $n-1$ functions $\psi^{(1)}, \ldots, \psi^{(n-1)}$, one for each node of 
the $A_{n-1}$ Dynkin diagram, should be introduced. These auxiliary functions are solutions of generally more-complicated ordinary differential equations.

Following [11, all the functions $\psi^{(a)}(x, E, \mathbf{g})$ decaying at large $x$ on the positive real axis can be constructed from $\psi \equiv \psi^{(1)}$ as

$$
\psi^{(a)}=W^{(a)}\left[\frac{1-a}{2}, \frac{3-a}{2}, \ldots, \frac{a-1}{2}\right] \equiv W^{(a)}\left[\psi_{\frac{1-a}{2}}, \psi_{\frac{3-a}{2}}, \ldots, \psi_{\frac{a-1}{2}}\right],
$$

where $a=1,2, \ldots, n-1, W^{(a)}\left[f_{1}, \ldots, f_{a}\right]$ denotes the generalised Wronskian of the set of functions $\left\{f_{a}\right\}$

$$
W^{(a)}\left[f_{1}, \ldots, f_{a}\right]=\operatorname{det}\left[\left(\vec{f}, \frac{d}{d x} \vec{f}, \ldots, \frac{d^{a-1}}{d x^{a-1}} \vec{f}\right)\right]
$$

with $\vec{f}=\left(f_{1}, f_{2}, \ldots, f_{a}\right)$ (so that $W^{(2)}[f, g] \equiv W[f, g]$, cf. eq. (3.33) ), and $\psi_{k}$ denotes the 'rotated' solution (3.29).

Finally, normalising $\psi^{(1)}$ by

$$
\mathcal{N}=\frac{i^{(n-1) / 2}}{\sqrt{n}} \Longrightarrow \psi^{(n)}(x, E, \mathbf{g})=1
$$

Since $\psi$ is a solution of an $n^{\text {th }}$-order ODE, a naive counting of degrees of freedom shows that the order of the ODE satisfied by $\psi^{(a)}$ should be

$$
\frac{n !}{(n-a) ! a !} \text {. }
$$

The $(n-a+1)$ and the $(a)$ equations are related by a $\left(\mathbf{g} \leftrightarrow \mathbf{g}^{\dagger}\right)$-conjugation [9, 11], arising from the $\mathbb{Z}_{2}$ symmetry of the Dynkin diagram. Notice that the result (4.14) exactly matches the dimensions of the basic representations of $A_{n-1}$; these are again in one-to-one correspondence with the nodes of the Dynkin diagram.

Fortunately, in order to derive the Bethe ansatz equations an explicit knowledge of the remaining $n-2$ ODEs is unnecessary: the derivation of [11] was instead based on the Stokes relation associated to (4.1)

$$
\sum_{k=0}^{n}(-1)^{k} C^{(k)}(E, \mathbf{g}) y_{k}(x, E, \mathbf{g})=0,
$$

where, according to [11],

$$
y_{k}(x, E, \mathbf{g})=\omega^{(n-1) k / 2} \psi\left(\omega^{-k} x, \Omega^{-k} E, \mathbf{g}\right),
$$

$C^{(0)}(E, \mathbf{g})=1$ and the Stokes multipliers $C^{(k)}(E, \mathbf{g})$ with $k>0$ are analytic functions of $E$ and $\mathbf{g}$. Stokes relations for the $D_{n}, B_{n}$ and $C_{n}$ equations (3.19), (3.20) 
and (3.21) also exist, but we have encountered some subtle complications $\S$ in generalising the approach of [11] to these cases.

Instead, the strategy here is based on the conjectured validity of a simple 'universal' system of functional equations among the $\psi^{(a)}$ functions, which leads immediately to the Bethe ansatz equations and bypasses the analysis of Stokes relations.

We shall now prove the $A_{n-1} \psi$-system (3.32):

$$
\psi^{(a-1)} \psi^{(a+1)}=W\left[\psi_{-\frac{1}{2}}^{(a)}, \psi_{\frac{1}{2}}^{(a)}\right], \quad \psi^{(0)}=\psi^{(n)}=1 .
$$

The proof is based on the observation that determinants satisfy functional equations, in particular the so-called Jacobi identity

$$
\Delta \Delta[p, q \mid p, q]=\Delta[p \mid p] \Delta[q \mid q]-\Delta[p \mid q] \Delta[q \mid p] .
$$

Here, $\Delta$ is the determinant of an $(a+1) \times(a+1)$ matrix and $\Delta\left[p_{1}, p_{2} \mid q_{1}, q_{2}\right]$ denotes the determinant of the same matrix with the $p_{1,2}-$ th rows and $q_{1,2}-$ th columns removed.

In order to prove (4.17) we have to consider three different cases: $a=1$, $1<a<n-1$ and $a=n-1$. The $a=1$ case follows from the definition of $\psi^{(2)}$ given in (4.11). Equation (4.17) for $1<a<n-1$ follows from the following chain of identities

$$
\begin{aligned}
& \prod_{b}\left(\psi^{(b)}\right)^{A_{a b}}=W^{(a+1)}\left[-\frac{a}{2},-\frac{a-2}{2}, \ldots, \frac{a-2}{2}, \frac{a}{2}\right] W^{(a-1)}\left[-\frac{a-2}{2}, \ldots, \frac{a-2}{2}\right] \\
& \quad=(-1)^{(a-1)} W^{(a+1)}\left[-\frac{a-2}{2}, \ldots, \frac{a-2}{2},-\frac{a}{2}, \frac{a}{2}\right] W^{(a-1)}\left[-\frac{a-2}{2}, \ldots, \frac{a-2}{2}\right] \\
& \quad=(-1)^{(a-1)} \Delta \Delta[a, a+1 \mid a, a+1]
\end{aligned}
$$

where we have identified

$$
\Delta \equiv W^{(a+1)}\left[-\frac{a-2}{2}, \ldots, \frac{a-2}{2},-\frac{a}{2}, \frac{a}{2}\right]
$$

and

$$
\Delta[a, a+1 \mid a, a+1]=W^{(a-1)}\left[-\frac{a-2}{2}, \ldots, \frac{a-2}{2}\right] .
$$

This is nothing but the LHS of the Jacobi identity (4.18). Then an application

\footnotetext{
${ }^{\S}$ In the $D_{n}$ case these complications are probably a consequence of the fact that the ODEs associated to the $\mathbb{Z}_{2}$-conjugate nodes in the Dynkin diagram are somehow more fundamental than eq. (3.19). This latter equation is more naturally associated to the first node on the 'tail' of the diagram.
} 
of the Jacobi identity naturally proves (4.17) in the following way:

$$
\begin{gathered}
\prod_{b}\left(\psi^{(b)}\right)^{A_{a b}}=(-1)^{(a-1)}(\Delta[a \mid a] \Delta[a+1 \mid a+1]-\Delta[a \mid a+1] \Delta[a+1 \mid a]) \\
=(-1)^{(a-1)}\left(W^{\prime(a)}\left[-\frac{a-2}{2}, \ldots, \frac{a-2}{2}, \frac{a}{2}\right] W^{(a)}\left[-\frac{a-2}{2}, \ldots, \frac{a-2}{2},-\frac{a}{2}\right]\right. \\
\left.\quad-W^{\prime(a)}\left[-\frac{a-2}{2}, \ldots, \frac{a-2}{2},-\frac{a}{2}\right] W^{(a)}\left[-\frac{a-2}{2}, \ldots, \frac{a-2}{2}, \frac{a}{2}\right]\right) \\
=W^{\prime(a)}\left[-\frac{a-2}{2}, \ldots, \frac{a-2}{2}, \frac{a}{2}\right] W^{(a)}\left[-\frac{a}{2},-\frac{a-2}{2}, \ldots, \frac{a-2}{2}\right] \\
\quad-W^{\prime(a)}\left[-\frac{a}{2},-\frac{a-2}{2}, \ldots, \frac{a-2}{2}\right] W^{(a)}\left[-\frac{a-2}{2}, \ldots, \frac{a-2}{2}, \frac{a}{2}\right] \\
=\psi_{-\frac{1}{2}}^{(a)} \psi_{\frac{1}{2}}^{\prime(a)}-\psi_{-\frac{1}{2}}^{(a)} \psi_{\frac{1}{2}}^{(a)}=W\left[\psi_{-\frac{1}{2}}^{(a)}, \psi_{\frac{1}{2}}^{(a)}\right]
\end{gathered}
$$

Finally, for $a=n-1$ the previous calculation shows that

$$
\psi^{(n-2)} \psi^{(n)}=W\left[\psi_{-\frac{1}{2}}^{(n-1)}, \psi_{\frac{1}{2}}^{(n-1)}\right] .
$$

Choosing $\mathcal{N}=\frac{i^{(n-1) / 2}}{\sqrt{n}}$ gives $\psi^{(n)}=1$ and (4.17) is proved.

\subsection{The $A_{n-1}$ Bethe ansatz equations}

In this section we shall show that the BAEs are a simple consequence of the $\psi$-system. First, recall the alternative $\chi$-basis of solutions (3.22) and the formal ordering of table 3

$$
g_{i}<g_{j} \quad \forall i<j .
$$

These solutions are defined by their behaviour as $x \rightarrow 0$

$$
\chi_{i}(x, E, \mathbf{g}) \sim x^{\lambda_{i}}+O\left(x^{\lambda_{i}+n}\right) \quad, \quad \lambda_{i}=g_{i} .
$$

Next, expand $\psi(x, E, \mathbf{g})$ in the $\chi$-basis

$$
\psi(x, E, \mathbf{g})=\sum_{i=0}^{n-1} Q_{[i]}^{(1)}(E, \mathbf{g}) \chi_{i}(x, E, \mathbf{g}),
$$

and use the property

$$
\chi_{i}\left(\omega^{k} x, \Omega^{k} E, \mathbf{g}\right)=\omega^{k \lambda_{i}} \chi_{i}(x, E, \mathbf{g})
$$

to obtain

$$
\psi_{k}(x, E, \mathbf{g})=\sum_{i=0}^{n-1} Q_{[i]}^{(1)}\left(\Omega^{k} E, \mathbf{g}\right) \omega^{k \lambda_{i}} \chi_{i}(x, E, \mathbf{g}) .
$$

Now expanding the determinants in the determinants for this new basis leads to

$$
\begin{aligned}
\psi^{(a)}(x, E, \mathbf{g}) & =\sum_{\mathbf{i}}\left(\prod_{j} Q_{\left[i_{j}\right]}^{(1)}\left(\Omega^{j} E, \mathbf{g}\right) \omega^{j \lambda_{i_{j}}}\right) W^{(a)}\left[\chi_{i_{\frac{1-a}{2}}}, \ldots, \chi_{i_{\frac{a-1}{2}}}\right] \\
& =\sum_{\mathbf{i}}^{\prime} Q_{\left[i_{\frac{i_{-a}}{2}}, \ldots, i_{\frac{a-1}{2}}\right]}^{(a)}(E, \mathbf{g}) W^{(a)}\left[\chi_{\frac{i_{\frac{1-a}{2}}}{2}} \ldots, \chi_{i_{\frac{a-1}{2}}}\right]
\end{aligned}
$$


where $j=\frac{1-a}{2}, \frac{3-a}{2}, \ldots, \frac{a-1}{2}, \sum_{\mathbf{i}}$ denotes the sum from 0 to $n-1$ of the set $\left\{i_{j}\right\}$ while in $\sum_{\mathbf{i}}^{\prime}$ there is the additional constraint $0 \leq i_{\frac{1-a}{2}} \leq i_{\frac{3-a}{2}} \leq \cdots \leq i_{\frac{a-1}{2}}$.

A family of $x$-independent equations is obtained by identifying from the LHS and RHS of (4.17) the terms corresponding to the same power. It is possible apriori to identify from every determinant only the highest, second highest, lowest and second lowest orders. We shall extract the leading orders, though similar results can be obtained from the subdominant ones.

Setting

$$
Q_{k}^{(a)}(E, \mathbf{g})=Q_{[0, \ldots, a-1]}^{(a)}\left(\Omega^{k} E, \mathbf{g}\right), \quad \bar{Q}_{k}^{(a)}(E, \mathbf{g})=Q_{[0, \ldots, a-2, a]}^{(a)}\left(\Omega^{k} E, \mathbf{g}\right)
$$

we have

$$
\begin{aligned}
\psi_{k}^{(a)}(x, E, \mathbf{g})= & \omega^{k \alpha_{a}} Q_{k}^{(a)}(E, \mathbf{g}) W^{(a)}\left[\chi_{0}, \ldots, \chi_{a-1}\right]+ \\
& \omega^{k\left(\alpha_{a+1}-\lambda_{a-1}\right)} \bar{Q}_{k}^{(a)}(E, \mathbf{g}) W^{(a)}\left[\chi_{0}, \ldots, \chi_{a-2}, \chi_{a}\right]+\ldots .
\end{aligned}
$$

The orders of the first and the second terms in (4.31) are $\alpha_{a}-a(a-1) / 2$ and $\alpha_{a+1}-\lambda_{a-1}-a(a-1) / 2$ respectively, where

$$
\alpha_{a}=\sum_{i=0}^{a-1} \lambda_{i}
$$

Substituting (4.31) into (4.17), comparing the leading terms of both sides for small $x$ and using the relation

$$
\begin{aligned}
W^{(a+1)} W^{(a-1)} & =W\left[W^{(a)}, \widehat{W}^{(a)}\right], \\
W^{(a)} & :=W^{(a)}\left[\chi_{0}, \ldots, \chi_{a-1}\right], \quad \widehat{W}^{(a)}:=W^{(a)}\left[\chi_{0}, \ldots, \chi_{a-2}, \chi_{a}\right]
\end{aligned}
$$

(also proved through the Jacobi identity), we find

$$
Q^{(a+1)} Q^{(a-1)}=\omega^{\frac{1}{2}\left(\lambda_{a}-\lambda_{a-1}\right)} Q_{-\frac{1}{2}}^{(a)} \bar{Q}_{\frac{1}{2}}^{(a)}-\omega^{\frac{1}{2}\left(\lambda_{a-1}-\lambda_{a}\right)} Q_{\frac{1}{2}}^{(a)} \bar{Q}_{-\frac{1}{2}}^{(a)},
$$

$Q^{(0)}=1$. Finally, let $E_{i}^{(a)}$ be a zero of $Q^{(a)}(E, \mathbf{g})$. Evaluating the above equation at $E=\Omega^{1 / 2} E_{i}^{(a)}$ and at $E=\Omega^{-1 / 2} E_{i}^{(a)}$, and by dividing the two equations thus obtained, we find the $A_{n-1}$ Bethe Ansatz equations

$$
\prod_{b=1}^{n-1} \Omega^{B_{a b} \gamma_{b}} \frac{Q_{B_{a b}}^{(b)}\left(E_{i}^{(a)}\right)}{Q_{-B_{a b}}^{(b)}\left(E_{i}^{(a)}\right)}=-1, \quad i=0,1,2, \ldots
$$

where

$$
\gamma_{a}=\frac{2 K}{M h^{\vee}}\left(\sum_{i=0}^{a-1} g_{i}-a \frac{(n-1)}{2}\right) .
$$


Notice that in writing relation (4.35) we have used the identity

$$
\frac{K}{M h^{\vee}}\left(\lambda_{a}-\lambda_{a-1}\right)=-\sum_{b=1}^{n-1} B_{a b} \gamma_{b},
$$

the ordering (4.24) and imposed the constraint $\gamma_{0}=\gamma_{n}=0$.

As shown in table 4, for $K=1$ there is very good agreement between the IM results obtained from the solution of a suitable non-linear integral equation (NLIE) (see $\S 6$ in [11]) and the direct numerical solution of the ODE.

\begin{tabular}{|c|c|c|}
\hline Level & $A_{4}$ NLIE & ODE numerics \\
\hline$E_{0}^{(1)}$ & 14.0495626907 & 14.0495626922 \\
$E_{1}^{(1)}$ & 47.7146839354 & 47.7146839363 \\
$E_{2}^{(1)}$ & 95.1785845453 & 95.1785845456 \\
$E_{3}^{(1)}$ & 154.202021469 & 154.202021470 \\
$E_{4}^{(1)}$ & 223.483044292 & 223.483044292 \\
\hline
\end{tabular}

Table 4: Comparison of IM results $\left(A_{4}\right.$ NLIE) with the direct numerical solution of the $A_{4}$ ODE with $K=1, M=10 / 21$ and $\mathbf{g}=(0.2,1.02,2.3,3.421)$.

Table 5 also shows the good agreement between the IM results obtained using the spin-1 NLIEs [44, 45] and the exact solution of the ODE with $K=2$ (see $₫ 6.2$ ).

\begin{tabular}{|c|c|c|}
\hline Level & $A_{1}$ NLIE with $K=2$ & ODE numerics \\
\hline$E_{0}^{(1)}$ & $1.49259741085 \pm 1.60304589242 i$ & $1.49259741085 \pm 1.60304589242 i$ \\
$E_{1}^{(1)}$ & $2.31180377628 \pm 2.38537059826 i$ & $2.31180377628 \pm 2.38537059826 i$ \\
$E_{2}^{(1)}$ & $2.91183770898 \pm 2.97068128676 i$ & $2.91183770898 \pm 2.97068128676 i$ \\
$E_{3}^{(1)}$ & $3.40837129214 \pm 3.45880577384 i$ & $3.40837129216 \pm 3.45880577388 i$ \\
$E_{4}^{(1)}$ & $3.84143464742 \pm 3.88626414305 i$ & $3.84143464640 \pm 3.88626414641 i$ \\
\hline
\end{tabular}

Table 5: Comparison of IM results $\left(A_{1}\right.$ NLIE) with the exact solution of the $A_{1}$ ODE with $K=2, M=1$ and $g_{0}=0$. The set $\left\{2 E_{i}^{(1)}\right\}$ are the exact eigenvalues of the $B_{1}$ linear ODE of 6.2

\section{The $D_{n}$ models}

The $D_{n}$ pseudo-differential equation (3.19) is

$$
\left(D_{n}\left(\mathbf{g}^{\dagger}\right)\left(\frac{d}{d x}\right)^{-1} D_{n}(\mathbf{g})-\sqrt{P_{K}(x, E)}\left(\frac{d}{d x}\right) \sqrt{P_{K}(x, E)}\right) \psi(x, E, \mathbf{g})=0 .
$$


Following the $A_{n-1}$ example, we start from the solution $\psi(x, E, \mathbf{g})$ of (5.1) with asymptotic behaviour

$$
\psi(x, E, \mathbf{g}) \sim \mathcal{N} x^{-h^{\vee} M / 2} \exp \left(-\frac{x^{M+1}}{M+1}\right) \quad, \quad\left(M>K /\left(h^{\vee}-K\right)\right)
$$

as $x \rightarrow \infty$ on the positive real axis, and introduce the alternative basis of solutions (3.22)

$$
\chi_{i}(x, E, \mathbf{g}), \quad i=0,1, \ldots, 2 n-1
$$

characterised by their behaviour near the origin

$$
\chi_{i}(x, E, \mathbf{g}) \sim x^{\lambda_{i}}+O\left(x^{\lambda_{i}+2 n-1}\right) \quad, \quad \begin{cases}\lambda_{i}=g_{i}, & \text { for } i \leq n-1 \\ \lambda_{i}=h^{\vee}-g_{2 n-1-i}, & \text { for } i>n-1\end{cases}
$$

In (5.4) the parameters $\lambda_{i}$ represent the $2 n$ solutions of the indicial equation (see table 2) with the ordering

$$
g_{i}<g_{j} \leq h^{\vee} / 2, \quad \lambda_{i}<\lambda_{j}, \quad \forall i<j .
$$

\subsection{The $\psi$-system and the $D_{n}$ Bethe ansatz equations}

To extract the $D_{n}$ BAE, we start from

$$
\psi_{k}^{(1)}=\psi_{k}(x, E, \mathbf{g})=\psi\left(\omega^{k} x, \Omega^{k} E, \mathbf{g}\right),
$$

where $\Omega$ and $\omega$ are as defined in (3.28) and (3.29), and assume the validity, for a suitable value of the normalisation constant $\mathcal{N}$, of the $\psi$-system (3.32)

$$
\begin{aligned}
W\left[\psi_{-\frac{1}{2}}^{(a)}, \psi_{\frac{1}{2}}^{(a)}\right] & =\psi^{(a-1)} \psi^{(a+1)}, \quad a=1, \ldots, n-3 \\
W\left[\psi_{-\frac{1}{2}}^{(n-2)}, \psi_{\frac{1}{2}}^{(n-2)}\right] & =\psi^{(n-3)} \psi^{(n-1)} \psi^{(n)}, \\
W\left[\psi_{-\frac{1}{2}}^{(n-1)}, \psi_{\frac{1}{2}}^{(n-1)}\right] & =W\left[\psi_{-\frac{1}{2}}^{(n)}, \psi_{\frac{1}{2}}^{(n)}\right]=\psi^{(n-2)} .
\end{aligned}
$$

Equations (15.8) and Jacobi identity (5.8) used in reverse imply the following relations linking the remaining functions $\psi^{(a)}(x, E, \mathbf{g})$ to $\psi^{(1)}(x, E, \mathbf{g})$ :

$$
\begin{gathered}
\phi^{(a)} \equiv \psi^{(a)}=W^{(a)}\left[\psi_{\frac{1-a}{2}}, \psi_{\frac{3-a}{2}}, \ldots, \psi_{\frac{a-1}{2}}\right], \quad a=1, \ldots, n-2, \\
\phi^{(n-1)} \equiv \psi^{(n-1)} \psi^{(n)}=W^{(n-1)}\left[\psi_{\frac{2-n}{2}}, \psi_{\frac{4-n}{2}}, \ldots, \psi_{\frac{n-2}{2}}\right],
\end{gathered}
$$

and

$$
\phi^{(n)} \equiv \psi_{-\frac{1}{2}}^{(n-1)} \psi_{\frac{1}{2}}^{(n-1)}+\psi_{-\frac{1}{2}}^{(n)} \psi_{\frac{1}{2}}^{(n)}=W^{(n)}\left[\psi_{\frac{1-n}{2}}, \psi_{\frac{3-n}{2}}, \ldots, \psi_{\frac{n-1}{2}}\right] .
$$


Now notice that the auxiliary functions $\phi^{(a)}(x, E, \mathbf{g})$ defined in (5.8), (5.9) and (5.10) satisfy an $A$-type $\psi$-system

$$
\phi^{(a-1)} \phi^{(a+1)}=W\left[\phi_{-\frac{1}{2}}^{(a)}, \phi_{\frac{1}{2}}^{(a)}\right], \quad a=1, \ldots, n-1 .
$$

Therefore, the arguments applied in $\$ 4.3$ go through in the same way:

$$
\begin{aligned}
\phi_{k}^{(a)}(x, E, \mathbf{g})= & \omega^{k \alpha_{a}} \widehat{Q}_{k}^{(a)}(E, \mathbf{g}) W^{(a)}\left[\chi_{0}, \ldots, \chi_{a-1}\right]+ \\
& \omega^{k\left(\alpha_{a+1}-\lambda_{a-1}\right)} \bar{Q}_{k}^{(a)}(E, \mathbf{g}) W^{(a)}\left[\chi_{0}, \ldots, \chi_{a-2}, \chi_{a}\right]+\ldots
\end{aligned}
$$

$(a=1,2, \ldots, n-1)$. The orders of the first and the second terms in (5.12) are given by $\alpha_{a}-a(a-1) / 2$ and $\alpha_{a+1}-\lambda_{a-1}-a(a-1) / 2$ respectively, with $\alpha_{a}=$ $\sum_{i=0}^{a-1} \lambda_{i}$ and

$$
\widehat{Q}_{k}^{(a)}(E, \mathbf{g})=\widehat{Q}^{(a)}\left(\Omega^{k} E, \mathbf{g}\right), \quad \bar{Q}_{k}^{(a)}(E, \mathbf{g})=\bar{Q}^{(a)}\left(\Omega^{k} E, \mathbf{g}\right)
$$

are $\phi$-related spectral determinants.

Using equation (5.11) we establish the following identity among $\phi$-related spectral determinants

$$
\widehat{Q}^{(a+1)}(E) \widehat{Q}^{(a-1)}(E)=\omega^{\frac{1}{2}\left(\lambda_{a}-\lambda_{a-1}\right)} \widehat{Q}_{-\frac{1}{2}}^{(a)}(E) \bar{Q}_{\frac{1}{2}}^{(a)}(E)-\omega^{\frac{1}{2}\left(\lambda_{a-1}-\lambda_{a}\right)} \widehat{Q}_{\frac{1}{2}}^{(a)}(E) \bar{Q}_{-\frac{1}{2}}^{(a)}(E) ;
$$

which leads to

$$
\frac{\widehat{Q}_{-\frac{1}{2}}^{(a-1)}\left(E_{i}^{(a)}\right)}{\widehat{Q}_{\frac{1}{2}}^{(a-1)}\left(E_{i}^{(a)}\right)} \frac{\widehat{Q}_{1}^{(a)}\left(E_{i}^{(a)}\right)}{\widehat{Q}_{-1}^{(a)}\left(E_{i}^{(a)}\right)} \frac{\widehat{Q}_{-\frac{1}{2}}^{(a+1)}\left(E_{i}^{(a)}\right)}{\widehat{Q}_{\frac{1}{2}}^{(a+1)}\left(E_{i}^{(a)}\right)}=-\Omega^{\frac{\alpha}{2}\left(\lambda_{a}-\lambda_{a-1}\right)}
$$

with $a=1,2, \ldots, n-1$ and $\alpha=\frac{2 K}{M h^{v}}$. We then make the following identifications

$$
\begin{aligned}
\widehat{Q}^{(0)}(E, \mathbf{g}) & =Q^{(0)}(E, \mathbf{g})=1 \\
\widehat{Q}^{(a)}(E, \mathbf{g}) & =Q^{(a)}(E, \mathbf{g}), \quad(a=1, \ldots, n-1) \\
\widehat{Q}^{(n-1)}(E, \mathbf{g}) & =Q^{(n-1)}(E, \mathbf{g}) Q^{(n)}(E, \mathbf{g}) ; \\
\widehat{Q}^{(n)}(E, \mathbf{g}) & =Q_{-\frac{1}{2}}^{(n-1)}(E, \mathbf{g}) Q_{\frac{1}{2}}^{(n-1)}(E, \mathbf{g})
\end{aligned}
$$

which reflect the relations among the $\phi$ 's and the $\psi$ 's in the above. In (5.19) we have implicitly assumed

$$
\psi^{(n)}(x, E, \mathbf{g})=o\left(\psi^{(n-1)}(x, E, \mathbf{g})\right)
$$

as $x \rightarrow 0$ and also that (see the discussion in 95.2 )

$$
Q^{(n-1)}\left(E,\left\{g_{0}, \ldots, g_{n-2}, g_{n-1}\right\}\right) \equiv Q^{(n)}\left(E,\left\{g_{0}, \ldots, g_{n-2}, h^{\vee}-g_{n-1}\right\}\right) .
$$


Plugging relations (5.17), (5.18) and (5.19) into (5.15) and using (5.21) is it easy to check that (5.15) can be recast in the universal form (2.1)

$$
\prod_{b=1}^{n} \Omega^{B_{a b} \gamma_{b}} \frac{Q_{B_{a b}}^{(b)}\left(E_{i}^{(a)}, \gamma\right)}{Q_{-B_{a b}}^{(b)}\left(E_{i}^{(a)}, \gamma\right)}=-1, \quad i=0,1,2, \ldots
$$

where $B_{a b}$ is the $D_{n}$-related matrix defined according to (2.4) and we have imposed the extra condition

$$
\gamma_{n}-\gamma_{n-1}=n-1-g_{n-1}
$$

to fix the exact $\left\{g_{a}\right\} \leftrightarrow\left\{\gamma_{a}\right\}$ relation as given in table 3,

Condition (5.23) guarantees that when $g_{n-1}-n+1=0$ the operator $(d / d x)^{-1}$ in (5.1) acts directly on a $d / d x$ and the relevant equation reduces to an $(2 n-1)$ order ODE. When this occurs $\gamma_{n}=\gamma_{n-1}$ and so the pair of $\mathbb{Z}_{2}$-conjugate nodes of the Dynkin diagram are 'twisted' in exactly the same way. Further, a change of sign in the RHS of (5.23) swaps $\gamma_{n}$ and $\gamma_{n-1}$, a property that naturally reflects the presence of the $\mathbb{Z}_{2}$-symmetry in the $D_{n}$ Dynkin diagram. All these properties are confirmed by the analysis of $\$ 5.2$ and $\$ 5.3$ and by 12-digits of numerical agreement at $K=1$ with $\mathbf{g}=\{0,1, \ldots, n-1\}$,

$$
\gamma_{a}=\alpha\left(\frac{(a-1) a}{2}-a \frac{h^{\vee}}{2}\right), \quad \gamma_{n}=\gamma_{n-1}=-\frac{n h^{\vee}}{4}
$$

between NLIE and ODE results.

Table 6 shows the (still-excellent) agreement at $K=1$ away from the $\gamma_{n}=\gamma_{n-1}$ surface. Appropriate $K>1$ NLIEs are unknown but numerical results qualitatively reproduce the expected IM scenario of $₫ 2$. Further analytic support to the proposed ODE/IM correspondence for $D_{n}$ is given in $\$ 5.2, \$ 5.3$ and $\$ 5.4$ below.

\begin{tabular}{|c|c|c|}
\hline Level & $D_{4}$ NLIE & ODE numerics \\
\hline$E_{0}^{(1)}$ & 17.8625636061 & 17.8625636061 \\
$E_{1}^{(1)}$ & 50.2942213433 & 50.2942213430 \\
$E_{2}^{(1)}$ & 92.8267466445 & 92.8267466442 \\
$E_{3}^{(1)}$ & 143.348705065 & 143.348705065 \\
$E_{4}^{(1)}$ & 200.738324171 & 200.738324172 \\
\hline
\end{tabular}

Table 6: Comparison of IM results ( $D_{4}$ NLIE) with the direct numerical solution of the $D_{4}$ pseudo-differential equation with $K=1, M=1 / 3$ and $\mathbf{g}=(0.2,1.1,2.3,2.95)$. 


\subsection{Example 1: $D_{2} \sim A_{1} \oplus A_{1}$}

The $D_{2}$ algebra can be decomposed into a pair of independent $A_{1}$ algebras, mirroring an analogous factorisation in the BAE. In this section we shall prove that the solution $\psi(x, E, \mathbf{g})$ to (5.1) with $n=2$ is the product of two solutions of $A_{1}$ related ODEs.

We start from the general $D_{2}$ equation:

$$
\begin{array}{r}
\left(( \frac { d } { d x } + \frac { g _ { 0 } } { x } ) \left(\frac{d}{d x}\right.\right. \\
\left.+\frac{g_{1}-1}{x}\right)\left(\frac{d}{d x}\right)^{-1}\left(\frac{d}{d x}-\frac{g_{1}-1}{x}\right)\left(\frac{d}{d x}-\frac{g_{0}}{x}\right) \\
\left.-\sqrt{P_{K}(x, E)}\left(\frac{d}{d x}\right) \sqrt{P_{K}(x, E)}\right) \psi(x, E, \mathbf{g})=0 .
\end{array}
$$

Expanding and integrating by parts, we obtain an equivalent equation:

$$
\begin{aligned}
\left(-\frac{d^{3}}{d x^{3}}+\right. & 4 P\left(x, E, g^{\prime}\right) \frac{d}{d x}+2 \frac{d P}{d x}\left(x, E, g^{\prime}\right) \\
& \left.+\frac{\left(1-g_{0}\right)^{2}\left(1-g_{1}\right)^{2}}{x^{2}}\left(\frac{d}{d x}\right)^{-1} \frac{1}{x^{2}}\right) \psi(x, E, \mathbf{g})=0
\end{aligned}
$$

where for this subsection it is convenient to define $P(x, E, k)=\frac{1}{4}\left[\left(x^{2 M / K}-E\right)^{K}+\right.$ $\left.\frac{k}{x^{2}}\right]$, and $g^{\prime}=g_{0}^{2}-2 g_{0}+g_{1}^{2}-2 g_{1}+1$.

We now set $Z(x)=\chi_{1}(x) \chi_{2}(x)$, a product of the solutions of two $A_{1}\left(\operatorname{spin}-\frac{1}{2}\right)$ equations, which for general $\rho$ and $\sigma$ satisfy

$$
\begin{aligned}
\frac{d^{2}}{d x^{2}} \chi_{1}(x, E, \rho) & =P(x, E, \rho) \chi_{1}(x, E, \rho), \\
\frac{d^{2}}{d x^{2}} \chi_{2}(x, E, \sigma) & =P(x, E, \sigma) \chi_{2}(x, E, \sigma) .
\end{aligned}
$$

To show that $Z(x)$ satisfies equation (5.26), we differentiate and repeatedly use the $A_{1}$ equations to find

$$
\begin{aligned}
\frac{d^{3} Z}{d x^{3}}=2 \frac{d P}{d x}(x, & \left., \frac{\rho+\sigma}{2}\right) Z+2 P\left(x, E, \frac{\rho+\sigma}{2}\right) \frac{d Z}{d x}+2 P(x, E, \rho) \chi_{1} \frac{d \chi_{2}}{d x} \\
+ & 2 P(x, E, \sigma) \frac{d \chi_{1}}{d x} \chi_{2} .
\end{aligned}
$$

If we now define the Wronskian

$$
W=\chi_{1} \frac{d \chi_{2}}{d x}-\frac{d \chi_{1}}{d x} \chi_{2}
$$

and use

$$
\chi_{1} \frac{d \chi_{2}}{d x}=\frac{1}{2}\left(\frac{d Z}{d x}+W\right) \quad, \quad \frac{d \chi_{1}}{d x} \chi_{2}=\frac{1}{2}\left(\frac{d Z}{d x}-W\right)
$$


then (5.29) can be written as

$$
\frac{d^{3} Z}{d x^{3}}=2 \frac{d P}{d x}\left(x, E, \frac{\rho+\sigma}{2}\right) Z+4 P\left(x, E, \frac{\rho+\sigma}{2}\right) \frac{d Z}{d x}+\frac{\rho-\sigma}{4 x^{2}} W .
$$

In order to express $W$ in terms of $Z$ we differentiate, apply (5.27) and (5.28), and then integrate:

$$
\frac{d W}{d x}=\chi_{1} \frac{d^{2} \chi_{2}}{d x^{2}}-\frac{d^{2} \chi_{1}}{d x^{2}} \chi_{2}=\frac{\sigma-\rho}{4 x^{2}} Z \quad \rightarrow \quad W=(\sigma-\rho)\left(\frac{d}{d x}\right)^{-1} \frac{Z}{4 x^{2}} .
$$

The resulting equation for $Z$,

$$
\begin{aligned}
\left(-\frac{d^{3}}{d x^{3}}+4 P(x, E\right. & \left.\frac{\rho+\sigma}{2}\right) \frac{d}{d x}+2 \frac{d P}{d x}\left(x, E, \frac{\rho+\sigma}{2}\right) \\
& \left.-\frac{(\rho-\sigma)^{2}}{16 x^{2}}\left(\frac{d}{d x}\right)^{-1} \frac{1}{x^{2}}\right) Z(x, E, \rho, \sigma)=0,
\end{aligned}
$$

exactly matches equation (5.26) provided the following relations between $g_{0}$ and $g_{1}$, and $\rho$ and $\sigma$ hold:

$$
\begin{aligned}
\rho+\sigma & =2\left(g_{0}^{2}-2 g_{0}+g_{1}^{2}-2 g_{1}+1\right) \\
\frac{\rho-\sigma}{4} & =\left(g_{0}-1\right)\left(g_{1}-1\right)
\end{aligned}
$$

If $\rho=\sigma$ then either $g_{0}$ or $g_{1}$ has to be zero and the integral operator in the $D_{2}$ equation acts on a total derivative. This observation agrees with the discussion in $\$ 5.1$ about the relation between $(d / d x)^{-1}$ and an asymmetric choice of the twists $\gamma_{n}$ and $\gamma_{n-1}$.

\subsection{Example 2: $D_{3} \sim A_{3}$}

The BAE for $A_{3}$ and $D_{3}$ are the same under identification of Bethe roots. It is therefore interesting to discuss the exact correspondence between the two models at the level of the pseudo-differential equations. Actually, it was the study of this case that lead us to the general $D_{n}$-related equations.

We start from the observation that the solution $\psi$ of the $A_{3}$-related ODE

$$
\left(D_{4}(\mathbf{g})-P_{K}(x, E)\right) \psi(x, E, \mathbf{g})=0,
$$

is associated to the first node of the $A_{3}$ Dynkin diagram, while the solution of the $D_{3}$ equation

$$
\left(D_{3}\left(\overline{\mathbf{g}}^{\dagger}\right)\left(\frac{d}{d x}\right)^{-1} D_{3}(\overline{\mathbf{g}})-\tau \sqrt{P_{K}(x, E)}\left(\frac{d}{d x}\right) \sqrt{P_{K}(x, E)}\right) \phi(x, E, \overline{\mathbf{g}})=0
$$


is more naturally associated to the central node of the $D_{3}=A_{3}$ Dynkin diagram. (A constant factor $\tau$ has been included in eq. (5.38) to take into account the possibly-different normalisations for the $E$ parameters.)

Therefore we are looking for a relationship between $\phi(x, E, \overline{\mathbf{g}})$ and

$$
\psi^{(2)}(x, E, \mathbf{g})=W\left[\psi_{-\frac{1}{2}}^{(1)}, \psi_{\frac{1}{2}}^{(1)}\right] .
$$

For simplicity, we perform the calculation at $\mathbf{g}=\{0,1,2\}$. We set

$$
\psi^{(2)}(x, E)=[0,1]
$$

where we have introduced the shorthand notation

$$
[i, j]=\left(\frac{d^{i}}{d x^{i}} \psi_{-\frac{1}{2}}^{(1)}\right)\left(\frac{d^{j}}{d x^{j}} \psi_{\frac{1}{2}}^{(1)}\right)-\left(\frac{d^{j}}{d x^{j}} \psi_{-\frac{1}{2}}^{(1)}\right)\left(\frac{d^{i}}{d x^{i}} \psi_{\frac{1}{2}}^{(1)}\right)
$$

so that

$$
\frac{d}{d x}[i, j]=[i+1, j]+[i, j+1] \quad, \quad[i, i]=0,
$$

and

$$
\frac{d^{4}}{d x^{4}} \psi_{ \pm \frac{1}{2}}^{(1)}(x, E)=-P_{K}(x, E) \psi_{ \pm \frac{1}{2}}^{(1)}(x, E) .
$$

Taking derivatives five times and using the above equation we have

$$
\begin{aligned}
\psi^{(2)} & =[0,1], \frac{d}{d x} \psi^{(2)}=[0,2], \frac{d^{2}}{d x^{2}} \psi^{(2)}=[1,2]+[0,3], \\
\frac{d^{3}}{d x^{3}} \psi^{(2)} & =2[1,3]+[0,4]=2[1,3]-P_{K}[0,0]=2[1,3] \\
\frac{d^{4}}{d x^{4}} \psi^{(2)} & =2[2,3]+2[1,4]=2[2,3]+2 P_{K} \psi^{(2)}, \\
\frac{d^{5}}{d x^{5}} \psi^{(2)} & =2[2,4]+2 \frac{d}{d x}\left(P_{K} \psi^{(2)}\right)=2 P_{K} \frac{d}{d x} \psi^{(2)}+2 \frac{d}{d x}\left(P_{K} \psi^{(2)}\right),
\end{aligned}
$$

and therefore obtain the desired ODE

$$
\left(\frac{d^{5}}{d x^{5}}-2 \sqrt{P_{K}(x, E)} \frac{d}{d x} \sqrt{P_{K}(x, E)}\right) \psi^{(2)}(x, E)=0,
$$

which matches (5.38) at $\tau=2$.

We have also checked that the solution $\psi^{(1)}(x, E, \mathbf{g})$ of the more general $A_{3^{-}}$ related differential equation (5.37) leads to a function $\psi^{(2)}(x, E, \mathbf{g})=W\left[\psi_{-\frac{1}{2}}^{(1)}, \psi_{\frac{1}{2}}^{(1)}\right]$, which is the solution of

$$
\left(D_{3}\left(\overline{\mathbf{g}}^{\dagger}\right)\left(\frac{d}{d x}\right)^{-1} D_{3}(\overline{\mathbf{g}})-2 \sqrt{P_{K}(x, E)}\left(\frac{d}{d x}\right) \sqrt{P_{K}(x, E)}\right) \psi^{(2)}(x, E, \mathbf{g})=0 .
$$


As already seen in $\$ 5.2$, in order to recast the resulting equation in the factorised form (5.46) one has perform a number of integrations by parts.

The exact relation between the $A_{3}$ and $D_{3}$ sets of parameters is

$$
\begin{aligned}
& 2 g_{0}=1+\bar{g}_{0}+\bar{g}_{1}-\bar{g}_{2}, \\
& 2 g_{1}=1+\bar{g}_{0}-\bar{g}_{1}+\bar{g}_{2}, \\
& 2 g_{2}=1-\bar{g}_{0}+\bar{g}_{1}+\bar{g}_{2} .
\end{aligned}
$$

\subsection{Relationship with the sine-Gordon model}

The reader may have noticed that the sets of numbers $\left\{m_{a}\right\}$ for the $D_{n}$ and $B_{n}$ models summarised in table 1 match the mass spectra of the sine-Gordon model at particular values of the coupling constant. From the sine-Gordon point of view the $D_{n}$-related mass spectrum emerges at the reflectionless points where the scattering between the solitons becomes purely diagonal. This link between the sine-Gordon model, affine Toda field theories and perturbed coset CFTs has been discussed in various places [46, 47, 48, 49].

In this section we would like to point out that there is a simple connection between equation (5.1) taken at

$$
K=1, \quad M=1 /(n-1), \quad \mathbf{g}=\{0,1, \ldots, n-1\}
$$

and the Schrödinger problem associated, through the first instance of the ODE/IM correspondence [1, 6], to the CFT limit of the sine-Gordon model. We start from (5.1) with parameters (5.48):

$$
\left(-\frac{d^{2 n-1}}{d x^{2 n-1}}+\left(x^{2}-E\right) \frac{d}{d x}+x\right) \chi(x, E)=0
$$

and require $\chi(x, E)$ to be absolutely integrable on the full real line; this restricts the possible values taken by $E$ to a discrete set. Fourier transforming (5.49) yields

$$
\left(-\frac{d^{2}}{d k^{2}}-\frac{1}{k} \frac{d}{d k}+\left((-1)^{n} k^{2 n-2}-E\right)\right) \tilde{\chi}(k, E)=0,
$$

and replacing

$$
k \rightarrow i k, \quad E \rightarrow-E, \quad \tilde{\chi}(k, E) \rightarrow k^{-1 / 2} \tilde{\chi}(k, E)
$$

we finally find

$$
\left(-\frac{d^{2}}{d k^{2}}+k^{2 n-2}-\frac{1}{4 k^{2}}-E\right) \tilde{\chi}(k, E)=0 .
$$

Equation (5.52) exactly matches the ODE associated in [1, 6] to the reflectionless points of the untwisted sine-Gordon model at its $c=c_{\text {eff }}=1$ conformal point.

This simple observation gives extra support to the correctness of the $D_{n}$ proposal (5.1), and it leads naturally to the $B_{n}$ proposals discussed in the next section. 


\section{The $B_{n}$ models}

The discussion in $\$ 5.4$ of the link between the sine-Gordon and $D_{n}$ scattering theories at specific values of the parameters can be extended to the $B_{n}$ models 48 , 49]. This and further considerations led us to the ODE (3.20), which we repeat here:

$$
\left(D_{n}\left(\mathbf{g}^{\dagger}\right) D_{n}(\mathbf{g})+\sqrt{P_{K}(x, E)}\left(\frac{d}{d x}\right) \sqrt{P_{K}(x, E)}\right) \psi(x, E, \mathbf{g})=0 .
$$

The results of $\$ 5.1$ and $\$ 5.2$ suggest a link between the presence of the integral operator $(d / d x)^{-1}$ and the possibility of breaking the symmetry between the $n$ and $n-1$ nodes of the $D_{n}$ Dynkin diagram by choosing $\gamma_{n} \neq \gamma_{n-1}$ in the BAE. Therefore, in writing (6.1) we have omitted the integral operator $(d / d x)^{-1}$ of

(5.1) because, in contrast to the $D_{n}$ Dynkin diagrams, the $B_{n}$ diagrams have no $\mathbb{Z}_{2}$ symmetry.

The relevant solution $\psi(x, E, \mathbf{g})$ to (6.1) has the asymptotic $x \rightarrow \infty$ behaviour

$$
\psi(x, E, \mathbf{g}) \sim \mathcal{N} x^{-h^{\vee} M / 2} \exp \left(-\frac{x^{M+1}}{M+1}\right) \quad, \quad\left(M>K /\left(h^{\vee}-K\right)\right)
$$

on the positive real axis. The solutions (3.22)

$$
\chi_{i}(x, E, \mathbf{g}) \quad, \quad i=0,1, \ldots, 2 n-1
$$

are instead characterised by the $x \rightarrow 0$ behaviour

$$
\chi_{i}(x, E, \mathbf{g}) \sim x^{\lambda_{i}}+O\left(x^{\lambda_{i}+2 n}\right), \quad\left\{\begin{array}{ll}
\lambda_{i}=g_{i}, & \text { for } i \leq n-1 \\
\lambda_{i}=h^{\vee}-g_{2 n-1-i}, & \text { for } i>n-1
\end{array} .\right.
$$

In (6.4) the $\lambda$ 's represent the $2 n$ solutions of the indicial equation in table 2 with the ordering

$$
g_{i}<g_{j}<h^{\vee} / 2, \quad \lambda_{i}<\lambda_{j}, \quad \forall i<j .
$$

\subsection{The $\psi$-system and the $B_{n}$ Bethe ansatz equations}

The $B_{n} \psi$-system is

$$
\begin{gathered}
\psi^{(a-1)} \psi^{(a+1)}=W\left[\psi_{-\frac{1}{2}}^{(a)}, \psi_{\frac{1}{2}}^{(a)}\right], \quad a=1, \ldots, n-1, \\
\psi_{-\frac{1}{4}}^{(n-1)} \psi_{\frac{1}{4}}^{(n-1)}=W\left[\psi_{-\frac{1}{4}}^{(n)}, \psi_{\frac{1}{4}}^{(n)}\right] .
\end{gathered}
$$

Using the identity (4.18) we can express $\psi^{(a)}$ with $a>1$ in terms of $\psi^{(1)} \equiv \psi$. The result is

$$
\psi^{(a)}=W\left[\psi_{\frac{1-a}{2}}, \ldots, \psi_{\frac{a-1}{2}}\right], \quad a=1,2, \ldots, n .
$$


Following the derivation in $₫ 4.3$, define $\alpha_{a}=\sum_{i=0}^{a-1} \lambda_{i}$,

$$
Q_{k}^{(a)}(E, \mathbf{g})=Q_{[0, \ldots, a-1]}^{(a)}\left(\Omega^{k} E, \mathbf{g}\right), \quad \bar{Q}_{k}^{(a)}(E, \mathbf{g})=Q_{[0, \ldots, a-2, a]}^{(a)}\left(\Omega^{k} E, \mathbf{g}\right)
$$

where $Q_{[0, \ldots, a-1]}^{(a)}$ and $Q_{[0, \ldots, a-2, a]}^{(a)}$ are defined as in (4.29) and

$$
\begin{aligned}
\psi_{k}^{(a)}(x, E, \mathbf{g})= & \omega^{k \alpha_{a}} Q_{k}^{(a)}(E, \mathbf{g}) W^{a}\left[\chi_{0}, \ldots, \chi_{a-1}\right]+ \\
& \omega^{k\left(\alpha_{a+1}-\lambda_{a-1}\right)} \bar{Q}_{k}^{(a)}(E, \mathbf{g}) W^{a}\left[\chi_{0}, \ldots, \chi_{a-2}, \chi_{a}\right]+\ldots
\end{aligned}
$$

Using (6.10) in (6.6) and identifying the leading contributions about $x=0$ gives

$$
Q^{(a+1)}(E) Q^{(a-1)}(E)=\omega^{\frac{1}{2}\left(\lambda_{a}-\lambda_{a-1}\right)} Q_{-\frac{1}{2}}^{(a)}(E) \bar{Q}_{\frac{1}{2}}^{(a)}(E)-\omega^{\frac{1}{2}\left(\lambda_{a-1}-\lambda_{a}\right)} Q_{\frac{1}{2}}^{(a)}(E) \bar{Q}_{-\frac{1}{2}}^{(a)}(E)
$$

with $Q^{(0)}(E)=1$ and

$$
\frac{Q_{-\frac{1}{2}}^{(a-1)}\left(E_{i}^{(a)}\right)}{Q_{\frac{1}{2}}^{(a-1)}\left(E_{i}^{(a)}\right)} \frac{Q_{1}^{(a)}\left(E_{i}^{(a)}\right)}{Q_{-1}^{(a)}\left(E_{i}^{(a)}\right)} \frac{Q_{-\frac{1}{2}}^{(a+1)}\left(E_{i}^{(a)}\right)}{Q_{\frac{1}{2}}^{(a+1)}\left(E_{i}^{(a)}\right)}=-\Omega^{-2 \gamma_{a}+\gamma_{a-1}+\gamma_{a+1}}
$$

In (6.12), $a=1, \ldots, n-1$ and

$$
\gamma_{a}=\alpha\left(\sum_{i=0}^{a-1} \lambda_{i}+a v\right), \quad a=1,2, \ldots, n,
$$

where $\alpha=\frac{2 K}{M h^{v}}$ and $v$ is still to be fixed. Plugging (6.10) into (6.7) leads to

$$
Q_{-\frac{1}{4}}^{(n-1)}(E) Q_{\frac{1}{4}}^{(n-1)}(E)=\omega^{\frac{1}{4}\left(\lambda_{n}-\lambda_{n-1}\right)} Q_{-\frac{1}{4}}^{(n)}(E) \bar{Q}_{\frac{1}{4}}^{(n)}(E)-\omega^{\frac{1}{4}\left(\lambda_{n-1}-\lambda_{n}\right)} Q_{\frac{1}{4}}^{(n)}(E) \bar{Q}_{-\frac{1}{4}}^{(n)}(E)
$$

and

$$
\frac{Q_{-\frac{1}{2}}^{(n-1)}\left(E_{i}^{(n)}\right)}{Q_{\frac{1}{2}}^{(n-1)}\left(E_{i}^{(n)}\right)} \frac{Q_{\frac{1}{2}}^{(n)}\left(E_{i}^{(n)}\right)}{Q_{-\frac{1}{2}}^{(n)}\left(E_{i}^{(n)}\right)}=-\Omega^{-\gamma_{n}+\frac{1}{2}\left(\gamma_{n-1}+\gamma_{n+1}\right)} .
$$

The boundary condition $\gamma_{n+1}=\gamma_{n-1}$ fixes $v=-h^{\vee} / 2$, the $\left\{g_{a}\right\} \leftrightarrow\left\{\gamma_{a}\right\}$ relation in table 3 and allows (6.12) and (6.15) to be recast in the form (2.1).

We have checked the consistency of the $n=2$ case both numerically and analytically. The relation with the sine-Gordon model briefly mentioned at the beginning of $₫ 6$ and the analysis of $₫ 6.2$ and $\$ 7.1 .2$ lend extra analytic support to the proposal. 


\subsection{Example 3: $B_{1}$}

This is a singular limit for the analytic BAE study in [37]. It however suggests the equivalence of the $K=1$ case of $B_{1}$ to the $K=2$ case of $A_{1}$ in the integrable system. Indeed, the differential equation is second order in the former case and it can be written in the more-standard form

$$
\left(\frac{d^{2}}{d x^{2}}+P_{K}(x, E) \frac{d}{d x}+\frac{1}{2}\left(\frac{d}{d x} P_{K}(x, E)\right)-\frac{g_{0}\left(g_{0}-1\right)}{x^{2}}\right) \psi\left(x, E, g_{0}\right)=0 \text {. }
$$

Performing a Liouville transformation

$$
\psi\left(x, E, g_{0}\right) \rightarrow \psi\left(x, E, g_{0}\right) \exp \left(-\frac{1}{2} \int^{x} P_{K}(\xi, E) d \xi\right)
$$

we find

$$
\left(-\frac{d^{2}}{d x^{2}}+\frac{1}{4}\left(x^{M / K}-E\right)^{2 K}+\frac{g_{0}\left(g_{0}-1\right)}{x^{2}}\right) \psi\left(x, E, g_{0}\right)=0 .
$$

Equation (6.18) coincides with the equations studied by Sergei Lukyanov 38] which are related to the $A_{1}$ lattice models with integer spin

$$
j=K, \quad K=1,2,3, \ldots .
$$

The cases $g_{0}=0$ with $M=K$ can be solved in closed form. After a shift $x \rightarrow$ $x+E$, the simplest case $K=1$ becomes

$$
\left(\frac{d^{2}}{d x^{2}}+x \frac{d}{d x}+\frac{1}{2}\right) \psi(x)=0
$$

which has general solution

$$
y(x)=c_{1} e^{-\frac{x^{2}}{2}} H_{-\frac{1}{2}}\left(\frac{x}{\sqrt{2}}\right)+c_{2} e^{-\frac{x^{2}}{4}} \sqrt{x} I_{-\frac{1}{4}}\left(\frac{x^{2}}{4}\right)
$$

where $H$ and $I$ are respectively the Hermite and the Bessel functions. Since

$$
H_{\nu}(x) \sim 2^{\nu} x^{\nu}(1+O(1 / x))
$$

as $\operatorname{Re}(x)>0,|x| \rightarrow \infty$, while

$$
I_{\nu}(x) \sim \frac{e^{x}}{\sqrt{2 \pi x}}(1+O(1 / x)),
$$

the most subdominant solution at large $x$ on the positive real axis is

$$
\psi(x)=e^{-\frac{x^{2}}{2}} H_{-\frac{1}{2}}\left(\frac{x}{\sqrt{2}}\right) .
$$

Figure 1 shows the first five complex-conjugate pairs of zeros of $\psi\left(-e^{\theta}\right)$. This 2 -string pattern is typical of $A_{1}$-related spin- 1 integrable models. The exact eigenvalues are reported in table 5 above. There is also good agreement between the position of the first pairs of zeros shown in figure 1 and the WKB asymptotic prediction of appendix $\mathrm{A}$. 


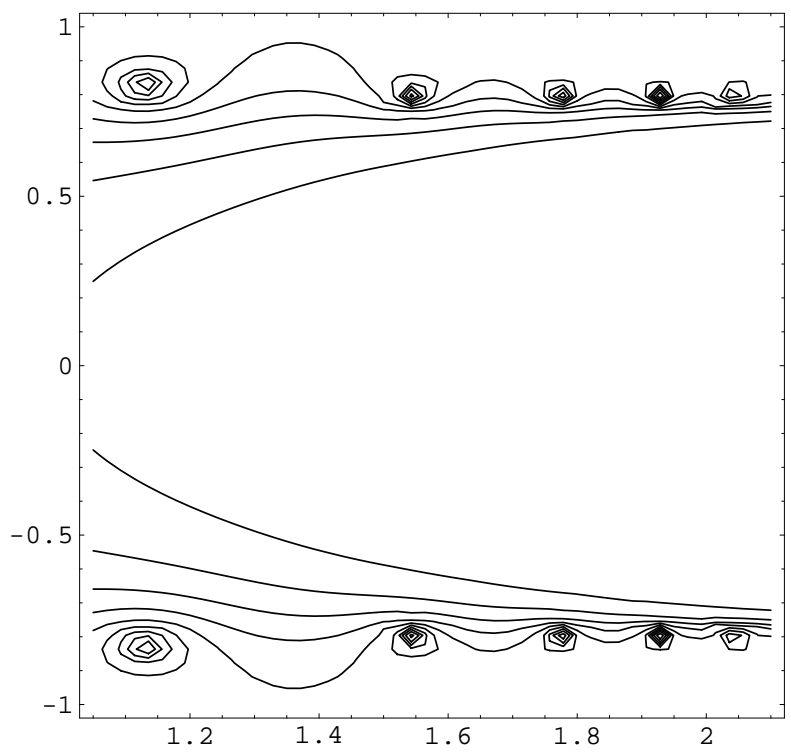

Figure 1: 2-strings in the $B_{1}$ model at $g_{0}=0, M=K=1$. Contour plot of $1 /(1+$ $\left.\left|H_{-\frac{1}{2}}\left(-\frac{1}{\sqrt{2}} e^{\theta}\right)\right|\right)$ in the complex $\theta$-plane.

\section{The $C_{n}$ models}

The analytic and numerical results of $\$ 5$ support the conjectured link between the $D_{n}$ BAE (2.1) and equation (5.1). At $\mathbf{g}=\{0,1, \ldots, n-1\}$, the $D_{n}$ ODE is

$$
\left(\frac{d^{2 n-1}}{d x^{2 n-1}}-\sqrt{P_{K}(x, E)}\left(\frac{d}{d x}\right) \sqrt{P_{K}(x, E)}\right) \psi(x, E)=0 .
$$

In this section, we start from (7.1) and consider a second duality relation

$$
\frac{\left(\widehat{D}_{-n}\right)_{K} \times\left(\widehat{D}_{-n}\right)_{L}}{\left(\widehat{D}_{-n}\right)_{K+L}} \sim \frac{\left(\widehat{C}_{n}\right)_{-K / 2} \times\left(\widehat{C}_{n}\right)_{-L / 2}}{\left(\widehat{C}_{n}\right)_{-K / 2-L / 2}}
$$

discussed by Hornfeck 41]. Motivated by the results of 4.1 on the analogy between the $A_{n} \leftrightarrow A_{-n}$ spectral duality and the $A_{n}$-related duality in [41], we change $n \rightarrow-n$ and $K \rightarrow-2 K$ in (7.1):

$$
\left((-1)^{-2 n-1} \frac{d^{-2 n-1}}{d x^{-2 n-1}}+\sqrt{P_{-2 K}(x, E)}\left(\frac{d}{d x}\right) \sqrt{P_{-2 K}(x, E)}\right) \psi(x, E)=0,
$$

where

$$
P_{-2 K}(x, E)=\left(x^{\frac{M(2 n+2)}{2 K}}-E\right)^{-2 K}
$$


Replacing

$$
\psi(x, E) \rightarrow\left(\left(P_{-2 K}(x, E)\right)^{-\frac{1}{2}}\left(\frac{d}{d x}\right)^{-1}\left(P_{-2 K}(x, E)\right)^{-\frac{1}{2}}\right) \psi(x, E),
$$

multiplying by $\frac{d^{2 n+1}}{d x^{2 n+1}}$ and noticing that

$$
\left(P_{-2 K}(x, E)\right)^{-\frac{1}{2}} \equiv P_{K}(x, E)=\left(x^{h^{\vee} M / K}-E\right)^{K},
$$

where $h^{\vee}=n+1$ is the dual Coxeter number of $C_{n}$, we can write the resulting equation as

$$
\left(\frac{d^{2 n+1}}{d x^{2 n+1}}-P_{K}(x, E)\left(\frac{d}{d x}\right)^{-1} P_{K}(x, E)\right) \psi(x, E)=0 .
$$

Equation (7.7) is our $C_{n}$ candidate at $\mathbf{g}=\mathbf{g}_{\mathbf{0}}=\{0,1,2, \ldots\}$. Further, adapting the discussion of $\$ 5$ that led to the full $B_{n}$ equation, and noting the similarity between the pseudo-differential operators (3.18), (3.19) and (3.20), we replace

$$
\frac{d^{2 n+1}}{d x^{2 n+1}} \equiv D_{n}\left(\mathbf{g}_{\mathbf{0}}^{\dagger}\right)\left(\frac{d}{d x}\right) D_{n}\left(\mathbf{g}_{0}\right) \Longrightarrow D_{n}\left(\mathbf{g}^{\dagger}\right)\left(\frac{d}{d x}\right) D_{n}(\mathbf{g})
$$

and the final $C_{n}$ proposal becomes (3.21):

$$
\left(D_{n}\left(\mathbf{g}^{\dagger}\right)\left(\frac{d}{d x}\right) D_{n}(\mathbf{g})-P_{K}(x, E)\left(\frac{d}{d x}\right)^{-1} P_{K}(x, E)\right) \psi(x, E, \mathbf{g})=0 .
$$

The relevant solution of (7.9) has the asymptotic $x \rightarrow \infty$ behaviour

$$
\psi(x, E, \mathbf{g}) \sim \mathcal{N} x^{-n M} \exp \left(-\frac{x^{M+1}}{M+1}\right) \quad, \quad\left(M>K /\left(h^{\vee}-K\right)\right)
$$

on the positive real axis. The solutions (3.22)

$$
\chi_{i}(x, E, \mathbf{g}), \quad i=0,1, \ldots, 2 n+1
$$

are characterised by the $x \rightarrow 0$ behaviour

$$
\chi_{i}(x, E, \mathbf{g}) \sim x^{\lambda_{i}}+O\left(x^{\lambda_{i}+2 n+1}\right) \quad, \quad \begin{cases}\lambda_{i}=g_{i}, & \text { for } i \leq n-1, \\ \lambda_{n}=n, & \\ \lambda_{i}=2 n-g_{2 n-i}, & \text { for } i>n\end{cases}
$$

In (7.12) the $\lambda$ 's represent the $2 n+1$ roots of the indicial equation in table 2 with the ordering

$$
g_{i}<g_{j}<n, \quad \lambda_{i}<\lambda_{j}, \quad \forall i<j
$$




\subsection{The $\psi$-system and the $C_{n}$ Bethe ansatz equations}

We now deduce the $C_{n}$ Bethe ansatz equations from the proposed $C_{n} \psi$-system:

$$
\begin{aligned}
W\left[\psi_{-\frac{1}{4}}^{(a)}, \psi_{\frac{1}{4}}^{(a)}\right] & =\psi^{(a-1)} \psi^{(a+1)}, \quad a=1,2, \ldots, n-2, \\
W\left[\psi_{-\frac{1}{4}}^{(n-1)}, \psi_{\frac{1}{4}}^{(n-1)}\right] & =\psi^{(n-2)} \psi_{-\frac{1}{4}}^{(n)} \psi_{\frac{1}{4}}^{(n)}, \\
W\left[\psi_{-\frac{1}{2}}^{(n)}, \psi_{\frac{1}{2}}^{(n)}\right] & =\psi^{(n-1)} .
\end{aligned}
$$

Using the Jacobi identity we find

$$
\begin{gathered}
\phi^{(a)} \equiv \psi^{(a)}=W^{(a)}\left[\psi_{\frac{1-a}{4}}, \psi_{\frac{3-a}{4}} \ldots, \psi_{\frac{a-1}{4}}\right], \quad a=1,2, \ldots, n-1, \\
\phi^{(n)} \equiv \psi_{-\frac{1}{4}}^{(n)} \psi_{\frac{1}{4}}^{(n)}=W^{(n)}\left[\psi_{\frac{1-n}{4}}, \psi_{\frac{3-n}{4}}, \ldots, \psi_{\frac{n-1}{4}}\right],
\end{gathered}
$$

where the functions $\phi^{(a)}(x, E, \mathbf{g})$ satisfy the following system of functional relations

$$
W\left[\phi_{-\frac{1}{4}}^{(a)}, \phi_{\frac{1}{4}}^{(a)}\right]=\phi^{(a-1)} \phi^{(a+1)}, \quad a=1, \ldots, n-1,
$$

and

$$
W\left[\phi_{-\frac{1}{4}}^{(n)}, \phi_{\frac{1}{4}}^{(n)}\right]=\phi^{(n-1)}\left(\psi^{(n)}\right)^{2} .
$$

From (7.18) we see that

$$
\psi^{(n)}(x, E, \mathbf{g})=\sqrt{W^{(n+1)}\left[\psi_{-\frac{n}{4}}, \ldots, \psi_{\frac{n}{4}}\right]} .
$$

Now set

$$
\begin{aligned}
\phi_{k}^{(a)}(x, E, \mathbf{g})= & \omega^{k \alpha_{a}} \widehat{Q}_{k}^{(a)}(E, \mathbf{g}) W^{(a)}\left[\chi_{0}, \ldots, \chi_{a-1}\right]+ \\
& \omega^{k\left(\alpha_{a+1}-\lambda_{a-1}\right)} \bar{Q}_{k}^{(a)}(E, \mathbf{g}) W^{(a)}\left[\chi_{0}, \ldots, \chi_{a-2}, \chi_{a}\right]+\ldots
\end{aligned}
$$

with $a=1,2, \ldots, n$. The orders of the first and the second term in (7.20) are given respectively by $\alpha_{a}-a(a-1) / 2$ and $\alpha_{a+1}-\lambda_{a-1}-a(a-1) / 2$, where $\alpha_{a}=\sum_{i=0}^{a-1} \lambda_{i}$. Assuming

$$
\psi_{k}^{(n)}(x, E, \mathbf{g})=\omega^{k \beta} x^{\beta} Q_{k}^{(n)}(E, \mathbf{g})+\omega^{k \sigma} x^{\sigma} \tilde{Q}_{k}^{(n)}(E, \mathbf{g})+\ldots
$$

where $\beta<\sigma$, we can make the following identifications

$$
\beta=\alpha_{n} / 2-n(n-1) / 4 \quad, \quad \sigma=\beta+\left(\lambda_{n}-\lambda_{n-1}\right)
$$

and

$$
\begin{aligned}
& \widehat{Q}^{(0)}(E)=Q^{(0)}(E)=1 \\
& \widehat{Q}^{(a)}(E)=Q^{(a)}(E), \quad a=1,2, \ldots, n-1, \\
& \widehat{Q}^{(n)}(E) \propto Q_{-\frac{1}{4}}^{(n)}(E) Q_{\frac{1}{4}}^{(n)}(E) \\
& \bar{Q}^{(n)}(E) \propto \omega^{\frac{1}{4}\left(\lambda_{n}-\lambda_{n-1}\right)} Q_{-\frac{1}{4}}^{(n)}(E) \tilde{Q}_{\frac{1}{4}}^{(n)}(E)+\omega^{\frac{1}{4}\left(\lambda_{n-1}-\lambda_{n}\right)} Q_{\frac{1}{4}}^{(n)}(E) \tilde{Q}_{-\frac{1}{4}}^{(n)}(E) .
\end{aligned}
$$


Using (17.20) in (7.17) and selecting the leading terms we find

$$
\widehat{Q}^{(a+1)}(E) \widehat{Q}^{(a-1)}(E)=\omega^{\frac{1}{4}\left(\lambda_{a}-\lambda_{a-1}\right)} \widehat{Q}_{-\frac{1}{4}}^{(a)}(E) \bar{Q}_{\frac{1}{4}}^{(a)}(E)-\omega^{\frac{1}{4}\left(\lambda_{a-1}-\lambda_{a}\right)} \widehat{Q}_{\frac{1}{4}}^{(a)}(E) \bar{Q}_{-\frac{1}{4}}^{(a)}(E) .
$$

With the identifications (7.24) and (7.25) this leads to

$$
\prod_{b=1}^{n} \frac{Q_{B_{a b}}^{(b)}\left(E_{i}^{(a)}\right)}{Q_{-B_{a b}}^{(b)}\left(E_{i}^{(a)}\right)}=-\Omega^{\frac{\alpha}{4}\left(\lambda_{a}-\lambda_{a-1}\right)}
$$

where $a=1,2, \ldots, n-1$ and $\alpha=\frac{2 K}{M h^{\mathrm{V}}}$. Using (7.20) with $a=n$ in (7.18), (7.26) and $(7.25)$ gives instead

$$
Q^{(n-1)}(E)=\omega^{\frac{1}{2}\left(\lambda_{n}-\lambda_{n-1}\right)} Q_{-\frac{1}{2}}^{(n)}(E) \tilde{Q}_{\frac{1}{2}}^{(n)}(E)-\omega^{\frac{1}{2}\left(\lambda_{n-1}-\lambda_{n}\right)} Q_{\frac{1}{2}}^{(n)}(E) \tilde{Q}_{-\frac{1}{2}}^{(n)}(E) ;
$$

which leads to

$$
\frac{Q_{-\frac{1}{2}}^{(n-1)}\left(E_{i}^{(n)}\right)}{Q_{\frac{1}{2}}^{(n-1)}\left(E_{i}^{(n)}\right)} \frac{Q_{1}^{(n)}\left(E_{i}^{(n)}\right)}{Q_{-1}^{(n)}\left(E_{i}^{(n)}\right)}=-\Omega^{\frac{\alpha}{2}\left(\lambda_{n}-\lambda_{n-1}\right)}
$$

Finally, with the identification (7.12) and the choice in table 3 for the $\left\{g_{a}\right\} \leftrightarrow\left\{\gamma_{a}\right\}$ relation, equations (7.28) and (7.30) can be assembled into the universal form (2.11).

\subsubsection{Example 4: $C_{1}$}

The $n=1$ case is again a singular limit of the analytic BAE, but it also suggests the similarity of this case to the $A_{1}$ models [37]. The pseudo-differential equation is, however, not second order but instead third order

$$
\left(\frac{d^{3}}{d x^{3}}-\frac{\mathcal{L}}{x^{2}} \frac{d}{d x}+\frac{\mathcal{L}}{x^{3}}-P_{K}(x, E)\left(\frac{d}{d x}\right)^{-1} P_{K}(x, E)\right) \psi\left(x, E, g_{0}\right)=0
$$

where $\mathcal{L}=g_{0}\left(g_{0}-2\right)$. It is nevertheless easy to check that (7.31) is solved by the product of two functions satisfying second order ODEs:

$$
\psi\left(x, E, g_{0}\right)=\chi_{-}\left(x, E, g_{0}\right) \chi_{+}\left(x, E, g_{0}\right)
$$

where $\chi_{ \pm}$originates from a single function $\chi$ as follows

$$
\chi_{ \pm}\left(x, E, g_{0}\right)=\chi\left(\omega^{ \pm 1 / 4} x, \Omega^{ \pm 1 / 4} E, g_{0}\right) .
$$

Since we assume that $\chi$ satisfies the standard ODE associated with $A_{1}$,

$$
\left(\frac{d^{2}}{d x^{2}}-\frac{1}{2} P_{K}(x, E)-\frac{\mathcal{L}}{4 x^{2}}\right) \chi\left(x, E, g_{0}\right)=0,
$$


the functions $\chi_{ \pm}$satisfy the following:

$$
\frac{d^{2}}{d x^{2}} \chi_{ \pm}\left(x, E, g_{0}\right)=\left( \pm \frac{i}{2} P_{K}(x, E)+\frac{\mathcal{L}}{4 x^{2}}\right) \chi_{ \pm}\left(x, E, g_{0}\right)
$$

Starting from (7.33) and differentiating $\psi=\psi\left(x, E, g_{0}\right)$ three times we find

$$
\begin{aligned}
\frac{d \psi}{d x} & =\frac{d \chi_{+}}{d x} \chi_{-}+\chi_{+} \frac{d \chi_{-}}{d x} ; \quad \frac{d^{2} \psi}{d x^{2}}=2 \frac{d \chi_{-}}{d x} \frac{d \chi_{+}}{d x}+\frac{\mathcal{L}}{2 x^{2}} \psi ; \\
\frac{d^{3} \psi}{d x^{3}} & =i P_{K}\left(\chi_{+} \frac{d \chi_{-}}{d x}-\frac{d \chi_{+}}{d x} \chi_{-}\right)+\frac{\mathcal{L}}{x^{2}} \frac{d \psi}{d x}-\frac{\mathcal{L}}{x^{3}} \psi .
\end{aligned}
$$

We notice that

$$
\frac{d}{d x}\left(\chi_{+} \frac{d \chi_{-}}{d x}-\frac{d \chi_{+}}{d x} \chi_{-}\right)=-i P_{K} \psi
$$

and therefore

$$
\chi_{+} \frac{d \chi_{-}}{d x}-\frac{d \chi_{+}}{d x} \chi_{-}=-i\left(\frac{d}{d x}\right)^{-1} P_{K} \psi .
$$

Inserting (7.38) into (7.36) we finally arrive at equation (7.31).

\subsubsection{Example 5: $C_{2} \sim B_{2}$}

The ODEs for $B_{2}$ and for $C_{2}$ are both deduced from the $D_{n}$-type ODE, but through completely different routes. It is thus a good test to check the equivalence of these two cases. We start with the $B_{2}$-related ODE at $\mathbf{g}=\{0,1\}$

$$
\frac{d^{4} \psi}{d x^{4}}+P_{K} \frac{d \psi}{d x}+\frac{1}{2} \frac{d P_{K}}{d x} \psi=0
$$

The ODE associated with the second node of $B_{2}$, which is nothing but the first node of $C_{2}$, would be satisfied by the following function

$$
\psi^{(2)}=W\left[\psi_{-\frac{1}{2}}, \psi_{\frac{1}{2}}\right]=[0,1],
$$

where

$$
\frac{d^{4} \psi_{ \pm \frac{1}{2}}}{d x^{4}}=P_{K} \frac{d \psi_{ \pm \frac{1}{2}}}{d x}+\frac{1}{2} \frac{d P_{K}}{d x} \psi_{ \pm \frac{1}{2}}
$$

We then easily evaluate the derivatives of $\psi^{(2)}$ :

$$
\begin{aligned}
\frac{d \psi^{(2)}}{d x} & =[0,2], \quad \frac{d^{2} \psi^{(2)}}{d x^{2}}=[1,2]+[0,3], \quad \frac{d^{3} \psi^{(2)}}{d x^{3}}=2[1,3]+P_{K} \psi^{(2)} \\
\frac{d^{4} \psi^{(2)}}{d x^{4}} & =2[2,3]+P_{K} \frac{d \psi^{(2)}}{d x}, \quad \frac{d^{5} \psi^{(2)}}{d x^{5}}=P_{K} \frac{d^{2} \psi^{(2)}}{d x^{2}}-2 P_{K}[1,2]
\end{aligned}
$$


Finally, noticing that

$$
2[1,2]=2\left(\frac{d}{d x}\right)^{-1}[1,3]=\frac{d^{2} \psi^{(2)}}{d x^{2}}-\left(\frac{d}{d x}\right)^{-1} P_{K} \psi^{(2)}
$$

we have obtained a closed form ODE for $\psi^{(2)}$ :

$$
\left(\frac{d^{5} \psi^{(2)}}{d x^{5}}-P_{K}(x, E)\left(\frac{d}{d x}\right)^{-1} P_{K}(x, E)\right) \psi^{(2)}(x, E)=0
$$

This equation is exactly the $\mathbf{g}=\{0,1\}$ equation associated to the first node of $C_{2}$. This verifies the consistency of our proposal.

More generally, the correspondence between the $B_{2}$ parameters $\mathbf{g}=\left\{g_{0}, g_{1}\right\}$ and the $C_{2}$ parameters $\mathbf{g}=\left\{\bar{g}_{0}, \bar{g}_{1}\right\}$ is established by

$$
\begin{aligned}
& 2 g_{0}=\bar{g}_{0}+\bar{g}_{1}-1, \\
& 2 g_{1}=\bar{g}_{0}-\bar{g}_{1}+3 .
\end{aligned}
$$

Finally we have directly checked the $C_{2}=B_{2} \psi$-system at $\mathbf{g}=\{0,1\}$ by verifying that both sides of

$$
W\left[\psi_{-\frac{1}{4}}^{(1)}, \psi_{\frac{1}{4}}^{(1)}\right]=\psi_{-\frac{1}{4}}^{(2)} \psi_{\frac{1}{4}}^{(2)},
$$

satisfy the same 15 th order ODE.

Unfortunately we do not currently have NLIEs for the $B_{n} / C_{n}$ models and the numerical checks presented below are instead based on an approximate solution similar to that used by Voros in [5]. For the $B_{2}$ BAEs for the case $\mathbf{g}=\{0,1\}, K=1$ and $M=2 / 3$, we started from a perfect-string estimate for the first 1000 roots as follows:

$$
\begin{array}{lr}
E_{j}=\left(4 \sqrt{\frac{\pi}{3}} \frac{\Gamma\left(\frac{11}{6}\right)}{\Gamma\left(\frac{4}{3}\right)} j\right)^{\frac{6}{5}} & \text { 1st node } \\
E_{j}=\mathrm{e}^{ \pm i \frac{\pi}{5}}\left(4 \sqrt{\pi} \frac{\Gamma\left(\frac{11}{6}\right)}{\Gamma\left(\frac{4}{3}\right)}\left(j-\frac{1}{6}\right)\right)^{\frac{6}{5}} & \text { 2nd node. }
\end{array}
$$

We then solved the BAEs recursively using the Newton-Raphson method on the first 20 roots, keeping the remaining roots fixed. Table 7 compares the lowest roots thus obtained with the results from the solution of the (pseudo-)differential equations. The relatively low accuracy in comparison with tables 4 , 5 and 6 is most likely to be a consequence of the slow convergence rate of the algorithm used to solve the Bethe ansatz equations, and in particular the systematic errors introduced by fixing the higher levels $\left(E_{j}, j>20\right)$ to their perfect-string values. 


\begin{tabular}{|ll|ll|}
\hline BA numerics & & ODE numerics & \\
1st node & 2nd node & 1st node & 2nd node \\
\hline 6.28405 & $6.8368 \pm 5.8640 i$ & 6.28390 & $6.8365 \pm 5.8637 i$ \\
13.2379 & $18.216 \pm 14.265 i$ & 13.2376 & $18.214 \pm 14.264 i$ \\
21.6307 & $30.996 \pm 23.645 i$ & 21.6303 & $30.992 \pm 23.642 i$ \\
30.5039 & $44.747 \pm 33.707 i$ & 30.5034 & $44.739 \pm 33.700 i$ \\
39.8617 & $59.254 \pm 44.304 i$ & 39.8613 & $59.240 \pm 44.292 i$ \\
\hline
\end{tabular}

Table 7: $C_{2}=B_{2}$ : comparison of Bethe ansatz results with the numerical solution of the $B_{2}$ and $C_{2}$ equations using the algorithm described in appendix $B$. $\left(B_{2}\right.$ node convention with $\mathbf{g}=\{0,1\}$, $K=1$ and $M=2 / 3$.)

\section{Conclusions}

There are many aspects about the correspondence between integrable models and the spectral theory of ordinary differential equations that we would like to explore and understand at a deeper level. Given the applications of the Bethe ansatz to the study of QCD in its leading-logarithm approximation [50] and to the study of anomalous dimensions of composite operators in Yang-Mills theories [51, 52, the extension of the correspondence to lattice models is certainly desirable from a physical point of view. On the other hand the mathematical structures arising from the generalisation of the correspondence to other conformal field theories with extended symmetries has the potential to link areas of modern and classical mathematics in an elegant way. In this paper our results were obtained very much on a case-by-case basis, but we already saw the emergence of interesting mathematical objects: the $\psi$-systems, negative-dimension dualities, and the formal similarity with the Miura-opers studied both in the classical work [19] (see also [53, 54, 55]) and more recently in [13, 14, 15, 17, 18].

It would be very interesting to generalise equations (3.18 3.21) to encompass the excited states of the integrable models; to date this has been completed for the $K=1$ case of $A_{1}$ [56, 57]. More challenging, but also extremely interesting, would be to extend the correspondence to perturbed conformal field theories defined on a cylinder, both for the ground state [58] and for excited states [59, 60].

Finally, even remaining inside the current setup, the ODE/IM correspondence has already had an impact on condensed matter physics: it has been applied to interacting Bose liquids [61, the single electron box [62] and quantum dots [63].

Acknowledgements - We are very grateful to Herman Boos, Boris Dubrovin, Ludwig Faddeev, Frank Goehmann, Andreas Klümper, Sergei Lukyanov and Fedor Smirnov for useful conversations and kind encouragement. PED, TCD and JS thank Torino University for hospitality at beginning of this project. JS also thanks the members of the Universities of Wuppertal and Bologna for hospitality and the Ministry of Education of Japan for a 'Grant-in-aid for Scientific 
Research', grant number 17540354. This project was also partially supported by the European network EUCLID (HPRN-CT-2002-00325), INFN grant TO12, NATO grant number PST.CLG.980424 and The Nuffield Foundation grant number NAL/32601, and a grant from the Leverhulme Trust.

\section{A Root strings from the complex WKB method}

As mentioned in 92 , a characteristic feature of the 'fused' models with $K>1$ is that the asymptotic roots are not necessarily real, but rather are grouped into so-called 'strings' of complex roots. Here we show how this behaviour can be recovered from a treatment of the ODE using the complex WKB method. We shall restrict the analysis to the $A_{1}$ case and for simplicity set $\mathbf{g}=\{0,1\}$. The ODE is the $n=2$ case of (4.1):

$$
\left(-\frac{d^{2}}{d z^{2}}+P_{K}(z)\right) \psi(z, E)=0, \quad P_{K}(z)=\left(z^{2 M / K}-E\right)^{K}
$$

and the boundary condition determining the eigenvalues is that there should be a solution which decays as $z \rightarrow \infty$ on the positive real axis, and is simultaneously zero at $z=0$. (In this section we use $z$ instead of $x$ to emphasise that it must be considered in the complex plane.)

Before giving the WKB treatment, we summarise the expectations from integrable models. A useful discussion of the $K=2$ case, for generic twist parameter, is in appendix 2 of [64]. For a finite lattice of size $N$, the integral equation derived in that paper leads to the following asymptotic condition on the Bethe ansatz roots $\theta_{j}^{ \pm}$

$$
N \ln \left(\tanh \left(\frac{\pi \theta_{j}^{ \pm}}{2 \gamma}\right)\right)= \pm N \pi i \mp\left(2 j-1+\frac{\phi}{\pi-2 \gamma}\right) \pi i+\frac{1}{2} \ln 2,
$$

where $1 \leq j<N / 2+1$. This equation describes the approximate position of complex-conjugate pairs of roots $\left\{\theta_{j}^{-}, \theta_{j}^{+}\right\}$, with $\theta_{j}^{-}$and $\theta_{j}^{+}$lying in the upper and lower halves of the complex plane. The relationship between $\gamma, \phi$ in (A.2) and $M$ and $\mathbf{g}=\left\{g_{0}, 1-g_{0}\right\}$ is

$$
\mu=\frac{M+1}{M}=\frac{\pi}{2 \gamma}, \quad \frac{\phi}{\pi-2 \gamma}=\frac{1}{2}-g_{0} .
$$

In the continuum limit, $N \rightarrow \infty$ and the numbers $e^{\theta_{j}^{ \pm}}$with $1 \leq j \ll N / 2$ tend to zero. Shifting $\theta_{j}^{ \pm}$appropriately, equation (A.2) becomes

$$
e^{2 \mu \theta_{j}^{ \pm}}= \pm\left(2 j-\frac{1}{2}-g_{0}\right) \pi i-\frac{1}{2} \ln 2, \quad j=1,2, \ldots .
$$


The second term causes deviations from the string pattern, even within this asymptotic approximation. Set $\theta_{j}^{ \pm}= \pm i \pi /(4 \mu)+\beta_{j}^{ \pm}$; then

$$
e^{2 \mu \beta_{j}^{ \pm}}=\left(2 j-\frac{1}{2}-g_{0}\right) \pi \pm \frac{i}{2} \ln 2
$$

or, taking logs and expanding for large $j$,

$$
2 \mu \beta_{j}^{ \pm}=\ln \left(2 j-\frac{1}{2}-g_{0}\right) \pi \pm i \frac{\ln 2}{2\left(2 j-\frac{1}{2}-g_{0}\right) \pi}+\ldots .
$$

Equation (A.6) exhibits the asymptotic deviations from the perfect string configurations, which are only recovered in the limit $j \rightarrow \infty$. This qualitative pattern matches the results of table 5 and figure 1, and is illustrated in figure 2, To compare with the $A_{1}, K=2$ results in $\$ 4.3$ one has to identify

$$
\left\{e^{2 \mu \theta_{j}^{+}}, e^{2 \mu \theta_{j}^{-}}\right\}=\left\{\mu^{-1}\left(E_{2 j-1}\right)^{\mu}, \mu^{-1}\left(E_{2 j}\right)^{\mu}\right\}
$$

in (A.4).

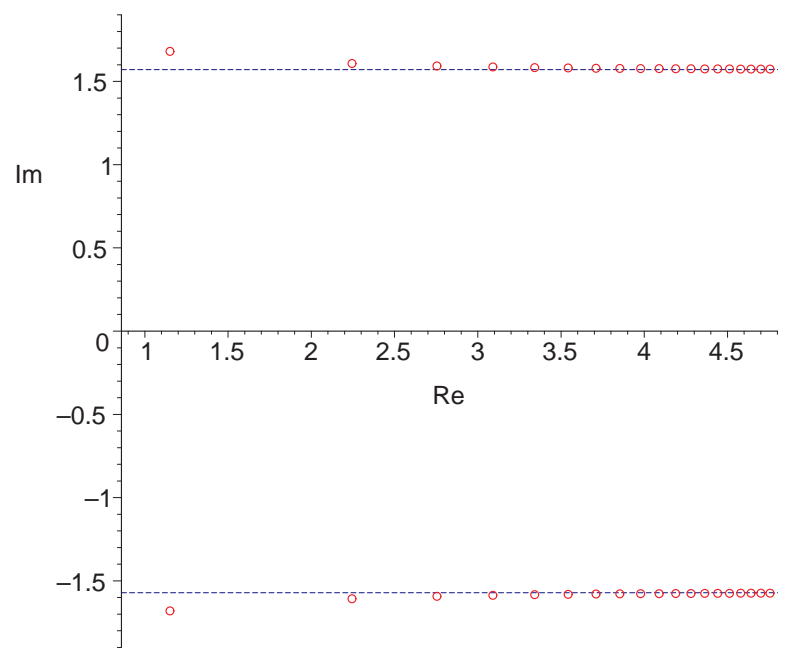

Figure 2: Two-strings from the asymptotics of the NLIE [64].

These features (and their generalisations to higher $K$ ) can all be recovered from a complex WKB treatment of (A.1). The practicalities of this method have been very clearly explained by Heading [65, 66], and we shall, more-or-less, follow his notations, which we now summarise. The behaviour of (A.1) is controlled by $P_{K}(z) \equiv\left(z^{2 M / K}-E\right)^{K}$, and the possibly-complex zeros of this function are called turning (or transition) points. For each turning point $z_{0}$, a branch cut is inserted joining $z_{0}$ to infinity; and for $2 M / K \notin \mathbb{Z}$, another is added starting 
from the origin along the negative-imaginary axis. After this, taking $\arg \left(P_{K}(z)\right)$ to tend to zero along the positive real $z$ axis renders $\left(P_{K}(z)\right)^{1 / 2}$ and $\left(P_{K}(z)\right)^{1 / 4}$ uniquely defined. From each turning point $z_{0}$ there is a set of lines

$$
\Im m \int_{z_{0}}^{z} d t \sqrt{P_{K}(t)}=0
$$

called Stokes lines, and another set

$$
\Re e \int_{z_{0}}^{z} d t \sqrt{P_{K}(t)}=0
$$

called anti-Stokes lines 7 . A $K^{\text {th }}$ order turning point has $K+2$ Stokes lines, and $K+2$ anti-Stokes lines, emanating from it. Leading-order WKB solutions are written as

$$
\left(z_{0}, z\right) \equiv\left(P_{K}(z)\right)^{-1 / 4} \exp \left(\int_{z_{0}}^{z} d t \sqrt{P_{K}(t)}\right) .
$$

If $\Re e \int_{z_{0}}^{z} d t \sqrt{P_{K}(t)}>0$, a subscript $d$ is added, signifying that $\left(z_{0}, z\right)_{d}$ is a dominant solution; $\left(z, z_{0}\right)$ is then subdominant, and is written $\left(z, z_{0}\right)_{s}$. On anti-Stokes lines, dominant and subdominant solutions swap rolesll.

Formal WKB solutions $\alpha\left(z, z_{0}\right)+\beta\left(z_{0}, z\right)$ are only valid in restricted domains: the Stokes phenomenon means that the coefficient of the subdominant term changes by an amount proportional to the dominant term when a Stokes line is crossed, so that $\alpha\left(z, z_{0}\right)_{d}+\beta\left(z_{0}, z\right)_{s}$ is replaced by $\alpha\left(z, z_{0}\right)_{d}+(\beta+T \alpha)\left(z_{0}, z\right)_{s}$, where the Stokes multiplier $T$ is a constant characteristic of the given Stokes line and crossing direction. (Note, since the discontinuity always occurs in the coefficient of a subdominant term, there is no contradiction with the fact that the WKB solution provides an asymptotic approximation to the exact - continuous solutions of the original equation.) For a Stokes line emanating from a $K^{\text {th }}$ order turning point, traversed in a positive (anticlockwise) sense, the Stokes multiplier is 67]

$$
T=2 i \cos (\pi /(K+2)) \text {. }
$$

This can alternatively be recovered via the $E=0, l=0, M=K / 2$ value of the function $C(E, l)$ discussed in [8], equation (3.10). For a clockwise traverse, $T$ is replaced by $-T$.

The key feature of (A.1), leading to the formation of strings, is that the turning points which control the WKB eigenvalues all have order $K$. If a solution

\footnotetext{
`Here we follow the conventions of Heading and, for example, Berry, but beware that other's conventions are exactly the reverse. Note also that our $P_{K}(z)$ is minus the function $q(z)$ used by Heading.

${ }^{\|}$Note, in contrast to elsewhere in this paper, the terms dominant and subdominant are used here with respect to the WKB expansion parameter, which for brevity we have set equal to 1 . The parameter, a factor of $\varepsilon^{2}$ in front of the derivative term in (A.1), can easily be restored if desired. In Heading's notations, $\varepsilon$ corresponds to $k^{-1}$.
} 
which decays along the positive real axis is traced in to a turning point, there are $K$ different directions away from that turning point along which the two WKB solutions $\left(z_{0}, z\right)$ and $\left(z, z_{0}\right)$ might come into balance and have a chance to cancel; if the continuation of any of these directions can be arranged to hit the origin, then a (WKB) eigenvalue is possible. When the Stokes multipliers have unit modulus, these directions are precisely the anti-Stokes lines; departures from unit modulus cause small shifts, and lead to deviations from the string pattern.

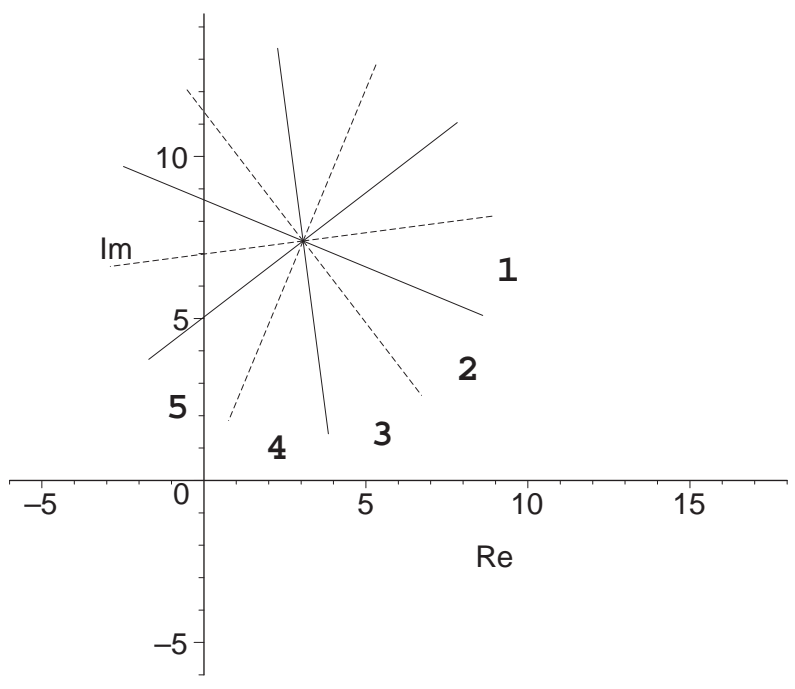

Figure 3: Initial directions of Stokes lines (continuous) and anti-Stokes lines (dotted) from a $4^{\text {th }}$ order turning point.

An example should make this clear. Figure 3 shows the set of Stokes and antiStokes directions away from a turning point of (A.1) with $M=3, K=4$. Between each pair of anti-Stokes lines there is a Stokes sector, which is further divided into two segments by the Stokes line which lies exactly in the middle of the Stokes sector. The first few of these segments are labelled $\mathbf{1} \ldots \mathbf{5}$ in the figure; note that segments $\mathbf{1}$ and $\mathbf{2}$ make up one Stokes sector, and segments $\mathbf{3}$ and $\mathbf{4}$ another. For the figure, $\arg (E)$ was chosen so that the origin lies in the union of segments 4 and $\mathbf{5}$, and the positive real axis in the union of the continuations of segments $\mathbf{1}$ and $\mathbf{2}$. Across the (dotted) anti-Stokes lines, dominant and subdominant solutions swap over, while across the (continuous) Stokes lines, the coefficients of subdominant terms may change by the Stokes phenomenon. Figure 4 shows the continuation of the Stokes directions shown in figure 3 into the full complex plane, justifying the claim that the real axis lies in the continuation of segments $\mathbf{1}$ and $\mathbf{2}$.

Our aim is to find an approximate solution which decays as $z \rightarrow \infty$ on the positive real axis, and is also zero at the origin. Since the positive real axis lies in the continuations of segments $\mathbf{1}$ and $\mathbf{2}$, we start with a solution subdominant 


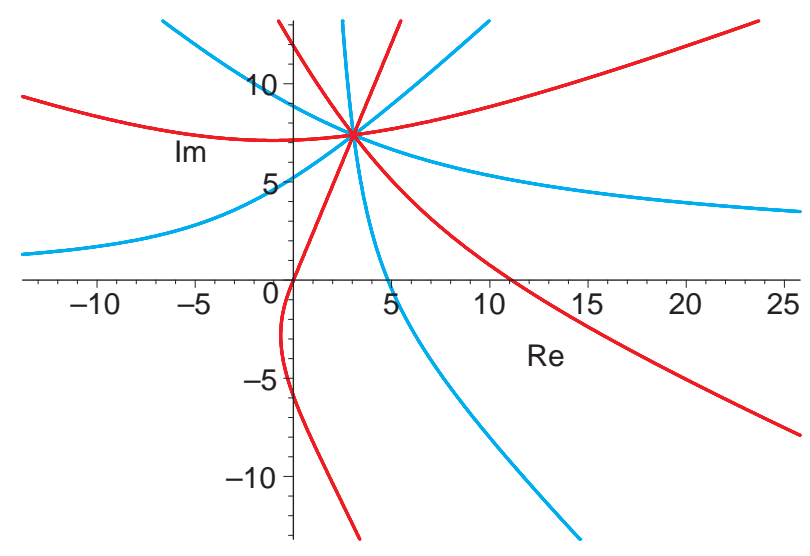

Figure 4: The full Stokes and anti-Stokes lines for the situation shown in figure 3 ,

in segments $\mathbf{1}$ and $\mathbf{2}$, and continue it clockwise around the turning point to find the behaviour in the region of the origin. There is no Stokes phenomenon passing between 1 to 2 since the dominant term is absent in both of these segments, but from then on the phenomenon must be taken into account each time a Stokes line is crossed. With $z_{0}$ the location of the turning point, and $\left(z, z_{0}\right)$ the approximate subdominant solution in segments $\mathbf{1}$ and $\mathbf{2}$, we have:

$$
\begin{aligned}
& \text { 1, 2: }\left(z, z_{0}\right)_{s} \\
& 3:\left(z, z_{0}\right)_{d} \\
& 4:\left(z, z_{0}\right)_{d}-2 i \cos (\pi / 6)\left(z_{0}, z\right)_{s} \\
& 5:\left(z, z_{0}\right)_{s}-2 i \cos (\pi / 6)\left(z_{0}, z\right)_{d}
\end{aligned}
$$

Near to the anti-Stokes line between segments $\mathbf{4}$ and $\mathbf{5}$, the WKB solutions $\left(z, z_{0}\right)$ and $2 i \cos (\pi / 6)\left(z_{0}, z\right)$ are of similar magnitude, and the (WKB) condition yielding the eigenvalues is found by demanding that they cancel exactly at $z=0$ :

$$
\left(z_{0}, 0\right)-2 i \cos (\pi / 6)\left(0, z_{0}\right)=0 \text {. }
$$

That is

$$
\exp \left(2 \int_{0}^{z_{0}} d t \sqrt{P_{K}(t)}+i \pi / 2\right)=\frac{1}{2 \cos (\pi / 6)}
$$

or

$$
2 \int_{0}^{z_{0}} d t \sqrt{P_{K}(t)}=\left(2 j-\frac{1}{2}\right) \pi i-\ln (2 \cos (\pi / 6)) .
$$

Remembering that $z_{0}=E^{K /(2 M)}$, the integral can be evaluated to

$$
\begin{aligned}
\int_{0}^{E^{K /(2 M)}}\left(t^{2 M / K}-E\right)^{K / 2} d t & =(-1)^{K / 2} E^{\mu} \int_{0}^{1}\left(1-u^{2 M / K}\right)^{K / 2} d u \\
& =(-1)^{K / 2} \tilde{\kappa}(2 M / K, 2 / K) E^{\mu}
\end{aligned}
$$


where $\mu=K(M+1) /(2 M)$ and

$$
\tilde{\kappa}(a, b)=\frac{\Gamma(1+1 / a) \Gamma(1+1 / b)}{\Gamma(1+1 / a+1 / b)} .
$$

Equations (A.14 A.16) give the WKB prediction at $K=4$. Had we instead performed the calculation for $K=2$, the logarithm on the RHS of (A.14) would have been replaced by $-\ln (2 \cos (\pi / 4))=-\frac{1}{2} \ln 2$, and (A.4) would have been reproduced. More generally, the Stokes multipliers solve a stationary T-system, and

the constant determining the deviation of the $j^{\text {th }}$ root in a $K$-string is found to be

$$
\ln (\sin (\pi j /(K+2)) / \sin (\pi(j+1) /(K+2))), \quad j=1,2, \ldots, K .
$$

The reason why only $K$ out of the $K+2$ anti-Stokes directions allow for zeros of the wavefunction is that on the two anti-Stokes lines next to the sector where the initial subdominant solution is defined (the anti-Stokes lines bordering the union of segments $\mathbf{1}$ and $\mathbf{2}$ in the example) there is only a single 'pure' WKB solution, and thus there is no chance to get the cancellation between two WKB solutions which leads to the wavefunction zeros near the other anti-Stokes directions.

It would be interesting to reproduce these results from a study of the relevant nonlinear integral equations in the integrable models, but we will leave a more detailed study of such issues for future work.

\section{B The numerical algorithm and the dual formulation of the boundary problem}

In this appendix we describe the numerical methods used to test our conjectures. We solved the pseudo-differential equations using an iterative power-series method, which we describe below. On the integrable model side, there is a wellknown method to rewrite an infinite set of Bethe ansatz equations such as (2.1) into a finite set of nonlinear integral equations (NLIEs) 68. We applied this to the $K=1$ simply-laced cases $A_{n-1}$ and $D_{n}$.

\section{B.1 The $\chi$-functions and the generalised Cheng algorithm.}

The special solution $\psi(x, E, \mathbf{g})$ and its derivatives can be written in terms of the $\chi$-functions (3.22) and the spectral determinants as

$$
\frac{d^{m}}{d x^{m}} \psi(x, E, \mathbf{g})=Q_{[0]}^{(1)}(E, \mathbf{g}) \frac{d^{m}}{d x^{m}} \chi_{0}(x, E, \mathbf{g})+Q_{[1]}^{(1)}(E, \mathbf{g}) \frac{d^{m}}{d x^{m}} \chi_{1}(x, E, \mathbf{g})+\ldots
$$


By solving for $Q_{[0]}^{(1)}(E, \mathbf{g})$ :

$$
Q_{[0]}^{(1)}(E, \mathbf{g})=\frac{W\left[\psi, \chi_{1}, \chi_{2}, \ldots\right]}{W\left[\chi_{0}, \chi_{1}, \chi_{2}, \ldots\right]} .
$$

In order to calculate the spectral determinant from (B.2) we must first find the $\chi$-solutions by numerically solving the pseudo-differential equations. This can be done, with very high precision, using a generalisation of the iteration method introduced by Cheng many years ago [20] and more recently used in [69]. In the following we shall define the iterative solution for each Lie algebra on a case-bycase basis.

$A_{n-1}$ : We begin by defining a solution for (3.18)

$$
D_{n}(\mathbf{g}) \chi(x, E, \mathbf{g})=(-1)^{n} P_{K}(x, E) \chi(x, E, \mathbf{g}),
$$

and its adjoint equation

$$
D_{n}\left(\mathbf{g}^{\dagger}\right) \chi^{\dagger}\left(x, E, \mathbf{g}^{\dagger}\right)=P_{K}(x, E) \chi^{\dagger}\left(x, E, \mathbf{g}^{\dagger}\right)
$$

where $\mathbf{g}=\left\{g_{n-1}, \ldots, g_{1}, g_{0}\right\}$ and $\mathbf{g}^{\dagger}=\left\{n-1-g_{0}, n-1-g_{1}, \ldots, n-1-g_{n-1}\right\}$.

Equation (B.4) was defined, as usual, by applying the rule $\left(d^{p} / d x^{p}\right)^{\dagger}=$ $(-1)^{p}\left(d^{p} / d x^{p}\right)$. Notice also that $(\underline{\text { B.4 }})$ is not the $\psi^{(n-1)}$-related ODE, which is instead obtained by simply replacing $\mathbf{g}$ with $\mathbf{g}^{\dagger}$ in $(\mathrm{B} .3)$.

Consider equation (B.3). The first step is to define a linear operator

$$
L_{\mathbf{g}}^{A}\left(x^{p}\right)=\frac{x^{p+n}}{\prod_{b=0}^{n-1}\left(p+n-g_{b}\right)}
$$

such that for any polynomial $\mathcal{P}(x)$ of $x$

$$
D_{n}(\mathbf{g}) L_{\mathbf{g}}^{A}(\mathcal{P}(x))=\mathcal{P}(x) .
$$

Now it is easy to check that

$$
\chi_{a}(x, E, \mathbf{g})=x^{g_{a}}+(-1)^{n} L_{\mathbf{g}}^{A}\left(P_{K}(x, E) \chi_{a}(x, E, \mathbf{g})\right)
$$

defines a solution to (B.3) solvable by iteration. Setting $g_{a}^{\dagger}=n-1-g_{n-1-a}$,

$$
\chi_{a}^{\dagger}\left(x, E, \mathbf{g}^{\dagger}\right)=x^{g_{a}^{\dagger}}+L_{\mathbf{g}^{\dagger}}^{A}\left(P_{K}(x, E) \chi_{a}^{\dagger}\left(x, E, \mathbf{g}^{\dagger}\right)\right),
$$

instead defines an iterative solution to the adjoint equation (B.4).

$D_{n}$ : The appropriate linear operator for the self-adjoint equation (3.19)

$$
D_{n}\left(\mathbf{g}^{\dagger}\right)\left(\frac{d}{d x}\right)^{-1} D_{n}(\mathbf{g}) \chi(x, E, \mathbf{g})=\sqrt{P_{K}(x, E)}\left(\frac{d}{d x}\right) \sqrt{P_{K}(x, E)} \chi(x, E, \mathbf{g})
$$


is

$$
L_{\mathbf{g}}^{D}\left(x^{p}\right)=\frac{\left(p+\frac{h^{\vee}}{2}+1\right) x^{p+h^{\vee}+1}}{\prod_{b=0}^{2 n-1}\left(p+1+\lambda_{b}\right)},
$$

and the corresponding iterative solution is

$$
\chi_{a}(x, E, \mathbf{g})=x^{\lambda_{a}}+L_{\mathbf{g}}^{D}\left(\sqrt{P_{K}(x, E)}\left(\frac{d}{d x}\right) \sqrt{P_{K}(x, E)} \chi_{a}(x, E, \mathbf{g})\right) .
$$

The relation between the $\lambda$ 's and the $g$ 's is given in (5.4).

$B_{n}$ : The iterative solution of the $B_{n}$ equation (3.20)

$$
D_{n}\left(\mathbf{g}^{\dagger}\right) D_{n}(\mathbf{g}) \chi(x, E, \mathbf{g})=-\sqrt{P_{K}(x, E)}\left(\frac{d}{d x}\right) \sqrt{P_{K}(x, E)} \chi(x, E, \mathbf{g})
$$

and its adjoint

$$
D_{n}\left(\mathbf{g}^{\dagger}\right) D_{n}(\mathbf{g}) \chi^{\dagger}(x, E, \mathbf{g})=\sqrt{P_{K}(x, E)}\left(\frac{d}{d x}\right) \sqrt{P_{K}(x, E)} \chi^{\dagger}(x, E, \mathbf{g})
$$

are, respectively,

$$
\chi_{a}(x, E, \mathbf{g})=x^{\lambda_{a}}-L_{\mathbf{g}}^{B}\left(\sqrt{P_{K}(x, E)}\left(\frac{d}{d x}\right) \sqrt{P_{K}(x, E)} \chi_{a}(x, E, \mathbf{g})\right)
$$

and

$$
\chi_{a}^{\dagger}(x, E, \mathbf{g})=x^{\lambda_{a}}+L_{\mathbf{g}}^{B}\left(\sqrt{P_{K}(x, E)}\left(\frac{d}{d x}\right) \sqrt{P_{K}(x, E)} \chi_{a}^{\dagger}(x, E, \mathbf{g})\right),
$$

with

$$
L_{\mathbf{g}}^{B}\left(x^{p}\right)=\frac{x^{p+h^{\vee}+1}}{\prod_{b=0}^{2 n-1}\left(p+1+\lambda_{b}\right)} .
$$

The relation between the $\lambda$ 's and the $g$ 's is given in (6.4).

$C_{n}$ : The $\chi$-solutions to the self-adjoint equation (3.21)

$$
D_{n}\left(\mathbf{g}^{\dagger}\right)\left(\frac{d}{d x}\right) D_{n}(\mathbf{g}) \chi_{a}(x, E, \mathbf{g})=P_{K}(x, E)\left(\frac{d}{d x}\right)^{-1} P_{K}(x, E) \chi_{a}(x, E, \mathbf{g})
$$

satisfy

$$
\chi_{a}(x, E, \mathbf{g})=x^{\lambda_{a}}+L_{\mathbf{g}}^{C}\left(P_{K}(x, E)\left(\frac{d}{d x}\right)^{-1} P_{K}(x, E) \chi_{a}(x, E, \mathbf{g})\right)
$$

with

$$
L_{\mathbf{g}}^{C}\left(x^{p}\right)=\frac{x^{p+2 n+1}}{\prod_{b=0}^{2 n-1}\left(p+1+\lambda_{b}\right)},
$$

and the relation between the $\lambda$ 's and the $g$ 's given in (7.12). 


\section{B.2 Dual formulation of the boundary problem}

From (B.2), with the numerical estimates for the $\chi$-functions and their derivatives at large values of $x$, replacing $\psi(x, E, \mathbf{g})$ by its asymptotic behaviour and by varying $E$ one has in principle access to $Q_{[0]}^{(1)}(E, \mathbf{g})$ and in particular to its zeros. This process is certainly possible but it is tedious and the CPU time increases at least quadratically with the order of the equation. Surprisingly, there is a short-cut that makes the algorithm essentially order-independent.

To see this, consider the $A_{n-1}$ case

$$
C Q_{[0]}^{(1)}(E, \mathbf{g})=W\left[\psi, \chi_{1}, \chi_{2}, \ldots, \chi_{n-1}\right]
$$

where

$$
C=W\left[\chi_{0}, \chi_{1}, \chi_{2}, \ldots, \chi_{n-1}\right]=(-1)^{\operatorname{int}[n / 2]} \prod_{i=0, j=i+1}^{n-1}\left(\lambda_{i}-\lambda_{j}\right) .
$$

Expand (B.20) with respect to the first column:

$$
C Q_{[0]}^{(1)}(E, \mathbf{g})=\sum_{p=0}^{n-1}(-1)^{p} w_{p} \frac{d^{p} \psi}{d x^{p}},
$$

where, borrowing the short-hand notation of $\$ 5.3$,

$$
\begin{aligned}
w_{p}(E, \mathbf{g}) & =[0,1, \ldots, p-1, p+1, \ldots, n-1] \\
& =\operatorname{det}\left[\left(\vec{\chi}, \frac{d}{d x} \vec{\chi}, \ldots, \frac{d^{p-1}}{d x^{p-1}} \vec{\chi}, \frac{d^{p+1}}{d x^{p+1}} \vec{\chi}, \ldots, \frac{d^{n-1}}{d x^{n-1}} \vec{\chi}\right)\right]
\end{aligned}
$$

with $\vec{\chi}=\left(\chi_{1}, \chi_{2}, \ldots, \chi_{n-1}\right)$. At large $x$ on the positive real axis

$$
\frac{d^{p}}{d x^{p}} \psi(x) \sim(-1)^{p} x^{(1-n+2 p) M / 2} \exp \left(-x^{M+1} /(M+1)\right) \quad, \quad\left(M>K /\left(h^{\vee}-K\right)\right) .
$$

Therefore, if for all functions $w_{p}(E, \mathbf{g})$ we have

$$
w_{p}\left(e_{i}, \mathbf{g}\right)=o\left(x^{(1-n) M / 2} \exp \left(x^{M+1} /(M+1)\right)\right), \quad p=0,1, \ldots, n-1
$$

for some $E=e_{i}$, then from (B.22)

$$
Q_{[0]}^{(1)}\left(e_{i}, \mathbf{g}\right)=0 .
$$

This shows that $\left\{e_{i}\right\} \subset\left\{E_{i}^{(1)}\right\}$. We shall now argue that the set $\left\{e_{i}\right\}$ is not empty and further prove that

$$
\left\{e_{i}\right\}=\left\{E_{i}^{(1)}\right\}
$$


To do this consider $w_{n-1}(E, \mathbf{g})=[0,1,3, \ldots, n-2]$. By repeated differentiation and substitution of the ODE, one can prove as in [11] that

$$
w_{n-1}(E, \mathbf{g})=\left((-1)^{\mathbf{i n t}[(n-1) / 2]} \prod_{i=1, j=i+1}^{n-1}\left(g_{i}-g_{j}\right)\right) \chi_{n-1}^{\dagger}\left(x, E, \mathbf{g}^{\dagger}\right)
$$

i.e. $w_{n-1}$ satisfies the adjoint $A_{n-1}$-equation (B.4) and thus can be determined using (B.8) with $g_{n-1}^{\dagger}=n-1-g_{0}$.

At large $x$,

$$
w_{n-1}(x)=S(E, \mathbf{g})\left(x^{(1-n) M / 2}+o\left(x^{(1-n) M / 2}\right)\right) \exp \left(\frac{x^{M+1}}{M+1}\right)+\ldots
$$

where the dots in (B.29) indicate terms that grow exponentially at most as

$$
\exp \left(\cos \left(\frac{2 \pi}{n}\right) \frac{x^{M+1}}{M+1}\right) \text {. }
$$

Therefore, the condition (B.25) for $p=n-1$ is fulfilled for any value of $E=e_{i}$ such that $S\left(e_{i}, \mathbf{g}\right)=0$. Since this a single condition imposed on a nontrivial function of $E$, we expect an infinite but countable set of solutions.

For the next step, we want to argue that if the condition (B.25) is satisfied for $p=n-1$ it is simultaneously satisfied for $p=0,1, \ldots, n-2$.

From (B.29) we see that at $S\left(e_{i}, \mathbf{g}\right)=0$ also

$$
\frac{d^{p}}{d x^{p}} w_{n-1}\left(e_{i}, \mathbf{g}\right)=o\left(x^{b} \exp \left(x^{M+1} /(M+1)\right)\right)
$$

for any finite complex number $b$. We now claim that all of the $w_{a}$ can be written as a linear combination of $w_{n-1}$ and its derivatives:

$$
w_{p}(x, E, \mathbf{g})=\sum_{a=0}^{n-1-p} \frac{K_{a}^{(p)}}{x^{n-1-p-a}} \frac{d^{a}}{d x^{a}} w_{n-1}(x, E, \mathbf{g})
$$

where the constants $K_{a}^{(p)}$ depend only on $\mathbf{g}$. It is very simple to see that (B.32) is true in general. Write the adjoint $A_{n-1}$ ODE in the 'expanded' form

$$
\frac{d^{n}}{d x^{n}} w_{n-1}=\left(\frac{A_{2}}{x^{2}} \frac{d^{n-2}}{d x^{n-2}}+\frac{A_{3}}{x^{3}} \frac{d^{n-3}}{d x^{n-3}}+\cdots+P_{K}(x, E)\right) w_{n-1} .
$$

Differentiating once

$$
\frac{d}{d x} w_{n-1}=[0,1,2, \ldots, n-3, n-1]=w_{n-2},
$$

taking further derivatives and using (B.33) the general result is

$$
w_{p-1}=\frac{d}{d x} w_{p}-\frac{A_{n-p}}{x^{n-p}} w_{n-1} .
$$


Solving (B.35) recursively proves (B.32).

Equation (B.32) and equation (B.31) together are equivalent to (B.25). To prove (B.27), substitute the asymptotic behaviours (B.24) and

$$
w_{n-1-i} \sim \frac{d^{i}}{d x^{i}} w_{n-1}(x) \sim S(E, \mathbf{g}) x^{(1-n+2 i) M / 2} \exp \left(\frac{x^{M+1}}{M+1}\right)
$$

into (B.22) to see that

$$
C Q_{[0]}^{(1)}(E, \mathbf{g})=n S(E, \mathbf{g}) \text {. }
$$

The eigenvalues are obtained by solving $\chi_{n-1}^{\dagger}\left(x, E, \mathbf{g}^{\dagger}\right)=0$ for fixed $x$, chosen large enough to capture the asymptotic behaviour of the solution while also ensuring the power series is reliable. This condition is different from the precise requirement (B.31), but considering (B.29) and (B.30) it is clear that, provided $x$ is very large, the error can be minimised and it selects the points on the complex $E$-plane where the function $S(E, \mathbf{g})$ is approximately zero.

Similarly, for $B_{n}$ the eigenvalues of the spectral determinant $Q_{[0]}^{(1)}$ are found by solving (B.15) for $\chi_{2 n-1}^{\dagger}(x, E, \mathbf{g})$. The situation is more complicated in the presence of the integral operators that appear in the $D_{n}$ and the $C_{n}$ models, and we have not yet fully completed the analysis. However, guided by the above and the results when the integral operator is absent, the obvious prescription to compute $\chi_{2 n-1}(x, E, \mathbf{g})$ from (B.11) and $\chi_{2 n+1}(x, E, \mathbf{g})$ from (B.18) for $D_{n}$ and $C_{n}$ respectively works very well.

\section{B.3 NLIEs}

The Bethe ansatz equations of type $A_{n-1}$ and $D_{n}$ for $K=1$ can be rephrased as a set of $r$ nonlinear integral equations where $r$ is the rank of the algebra [68] (see also [70, 64, 71, 3, 9, 11, 72, 73]). They are compactly written as

$$
\begin{aligned}
& f^{(a)}(\theta)=-\frac{2 i \pi}{h^{\vee}} \frac{\gamma_{a}}{\alpha}-2 i b_{0} e^{\theta} \\
& +\sum_{b=1}^{r}\left(\int_{\mathcal{C}_{1}} d \theta^{\prime} \varphi_{a b}\left(\theta-\theta^{\prime}\right) \ln \left(1+e^{f^{(b)}\left(\theta^{\prime}\right)}\right)-\int_{\mathcal{C}_{2}} d \theta^{\prime} \varphi_{a b}\left(\theta-\theta^{\prime}\right) \ln \left(1+e^{-f^{(b)}\left(\theta^{\prime}\right)}\right)\right),
\end{aligned}
$$

with $b_{0}=m_{1} \sin \mu$ and

$$
\varphi_{a b}(\theta)=\int_{-\infty}^{\infty} \frac{d k}{2 \pi} e^{i k \theta}\left(\delta_{a b}-\frac{\sinh (\pi \mu k)}{\sinh \left(\frac{\pi k}{h^{\vee}}\left(h^{\vee} \mu-1\right) k\right) \cosh \left(\frac{\pi k}{h^{\vee}}\right)} C_{a b}^{-1}(k)\right) .
$$

The integration contours $\mathcal{C}_{1}$ and $\mathcal{C}_{2}$ run from $-\infty$ to $\infty$ just below and just above the real axis respectively. The algebra dependence is encoded in the constant $b_{0}$, the 'twists' $\left\{\gamma_{a} / \alpha\right\}$, and the deformed Cartan matrix

$$
C_{a b}(k)=\left\{\begin{array}{ll}
2 & a=b \\
\frac{-1}{\cosh \left(\frac{\pi k}{h}\right)} & <a b>
\end{array} .\right.
$$


Above, $\langle a b\rangle$ implies the nodes $a$ and $b$ of the Dynkin diagram are connected. The explicit expressions for the inverse Cartan matrices can be found in 68].

By construction, the spectrum of the ordinary differential equation is encoded in the zeros $\theta_{j}$ of $1+\exp \left(f^{(a)}(\theta)\right.$ that lie on the real axis of the complex- $\theta$ plane [1]. The eigenvalues of the relevant ODE are obtained via the relation

$$
E_{j}=e^{\theta_{j} / \mu}
$$

The large- $E$ WKB-like behaviour can be obtained from the large- $\theta$ limit of these equations. In this limit the convolution terms in (B.39) can be dropped, and imposing $f^{(1)}\left(\theta_{j}\right)=i \pi(2 j-1)$ for integer $j$ yields the semiclassical prediction

$$
2 \sin (\mu \pi) m_{a}\left(E_{j}^{(a)}\right)^{\mu} \sim\left(2 j+1-\frac{2}{\alpha h^{\vee}} \gamma_{a}\right) \pi, \quad j=0,1,2, \ldots
$$

with $\alpha=2\left(\mu-1 / h^{\vee}\right)$.

\section{References}

[1] P. Dorey and R. Tateo, 'Anharmonic oscillators, the thermodynamic Bethe ansatz, and nonlinear integral equations', J. Phys. A 32, L419 (1999), hep-th/9812211.

[2] V.V. Bazhanov, S.L. Lukyanov and A.B. Zamolodchikov, 'Integrable structure of conformal field theory, quantum KdV theory and thermodynamic Bethe ansatz', Commun. Math. Phys. 177, 381 (1996), hep-th/9412229].

[3] V.V. Bazhanov, S.L. Lukyanov and A.B. Zamolodchikov, 'Integrable Structure of Conformal Field Theory II. Q-operator and DDV equation', Commun. Math. Phys. 190, 247 (1997), hep-th/9604044.

[4] Y. Sibuya, 'Global theory of a second-order linear ordinary differential equation with polynomial coefficient', (Amsterdam: North-Holland 1975).

[5] A. Voros, 'Semi-classical correspondence and exact results: the case of the spectra of homogeneous Schrödinger operators', J. Physique Lett. 43, L1 (1982);

- 'The return of the quartic oscillator. The complex WKB method', Ann. Inst. Henri Poincaré Vol XXXIX, 211 (1983);

- 'Exact resolution method for general 1D polynomial Schrödinger equation', J. Phys. A32, 5993 (1999) (and Corrigendum J. Phys. A 34, 5783 (2000)), math-ph/9903045.

[6] V.V. Bazhanov, S.L. Lukyanov and A.B. Zamolodchikov, 'Spectral determinants for Schroedinger equation and Q-operators of conformal field theory', J. Statist. Phys. 102, 567 (2001), hep-th/9812247. 
[7] J. Suzuki, 'Anharmonic oscillators, spectral determinant and short exact sequence of $U(q)(\widehat{s l}(2))$ ', J. Phys. A 32, L183 (1999), hep-th/9902053.

[8] P. Dorey and R. Tateo, 'On the relation between Stokes multipliers and the T-Q systems of conformal field theory', Nucl. Phys. B 563, 573 (1999) [Erratum-ibid. B 603, 581 (2001)], hep-th/9906219].

[9] P. Dorey and R. Tateo, 'Differential equations and integrable models: The SU(3) case', Nucl. Phys. B 571, 583 (2000) [Erratum-ibid. B 603, 582 (2001)], hep-th/9910102.

[10] J. Suzuki, 'Functional relations in Stokes multipliers and solvable models related to U(q) $\left(A_{n}^{(1)}\right)^{\prime}$, J. Phys. A 33, 3507 (2000), hep-th/9910215.

[11] P. Dorey, C. Dunning and R. Tateo, 'Differential equations for general SU(n) Bethe ansatz systems', J. Phys. A 33, 8427 (2000), hep-th/0008039.

[12] V.V. Bazhanov, A.N. Hibberd and S.M. Khoroshkin, 'Integrable structure of W(3) conformal field theory, quantum Boussinesq theory and boundary affine Toda theory', Nucl. Phys. B 622, 475 (2002), hep-th/0105177).

[13] E. Mukhin and A. Varchenko, 'Critical points of master functions and flag varieties', Commun. Contemp. Math. 6, 111 (2004), math.qa/0209017.

[14] E. Mukhin and A. Varchenko, 'Solutions to the XXX type Bethe ansatz equations and flag varieties', Cent. Eur. J. Math. 1, 238 (2003), math.qa/0211321].

[15] E. Mukhin and A. Varchenko, 'Populations of solutions of the XXX Bethe equations associated to Kac-Moody algebras', published in 'Infinite-dimensional aspects of representation theory and applications', Contemp. Math., 392, 95 (2005) Amer. Math. Soc., Providence, RI, math.qa/0212092].

[16] E. Frenkel, 'Opers on the projective line, flag manifolds and Bethe Ansatz', math.QA/0308269.

[17] E. Frenkel, 'Lectures on the Langlands program and conformal field theory', hep-th/0512172.

[18] A. Chervov and D. Talalaev, 'Quantum spectral curves, quantum integrable systems and the geometric Langlands correspondence', hep-th/0604128.

[19] V.G. Drinfel'd and V.V. Sokolov, 'Lie algebras and equations of Korteweg-de Vries type', J. Sov. Math. 30, 1975 (1984).

[20] H. Cheng, 'Meromorphic property of the S matrix in the complex plane of angular momentum', Phys. Rev. 127, 647 (1962).

[21] C.L. Schulz, 'Eigenvectors of multi-component generalization of the six-vertex model', Physica A 122, 71 (1983). 
[22] O. Babelon, H.J. de Vega and C.M. Viallet, 'Exact excitation spectrum of the $\mathrm{Z}(\mathrm{N}+1) \times \mathrm{Z}(\mathrm{N}+1)$ generalized Heisenberg model', Nucl. Phys. B 220, $283(1983)$.

[23] N.Y. Reshetikhin, 'Integrable models of quantum one-dimensional magnets with $\mathrm{O}(\mathrm{N})$ and $\mathrm{SP}(2 \mathrm{~K})$ symmetry', Theor. Math. Phys. 63, 555 (1985) [Teor. Mat. Fiz. 63 (1985) 347].

[24] N.Y. Reshetikhin, 'Algebraic Bethe ansatz for $\mathrm{SO}(\mathrm{n})$ invariant transfer-matrices', LOMI 169, 122 (1984) (in Russian).

[25] N.Y. Reshetikhin, 'The spectrum of the transfer matrices connected with Kac-Moody algebras', Lett. Math. Phys. 14, 235 (1987).

[26] N.Y. Reshetikhin and P.B. Wiegmann, 'Towards the classification of completely integrable quantum field theories', Phys. Lett. B 189, 125 (1987).

[27] P. Dorey, C. Dunning and R. Tateo, 'Aspects of the ODE/IM correspondence', in 'Recent trends in exponential asymptotics', RIMS Research Project 2004, Kyoto, Japan, hep-th/0411069.

[28] P.P. Kulish, N.Y. Reshetikhin and E.K. Sklyanin, 'Yang-Baxter Equation And Representation Theory: I', Lett. Math. Phys. 5, 393 (1981).

[29] V. Bazhanov and N. Reshetikhin, 'Restricted Solid On Solid models connected with simply laced algebras and conformal field theory', J. Phys. A 23, 1477 (1990).

[30] A. Kuniba, 'Thermodynamics of the $U_{q}\left(X_{r}^{(1)}\right)$ Bethe ansatz system with $q$ a root of unity', Nucl. Phys. B 389, 209 (1993).

[31] M. Takahashi, M. Suzuki, 'One-dimesional anisotropic Heisenberg model at finite temperature', Prog. Theoret. Phys. 48, 2187 (1972).

[32] A.B. Zamolodchikov, 'On the thermodynamic Bethe ansatz equations for reflectionless ADE scattering theories', Phys. Lett. B 253, 391 (1991).

[33] A. Kuniba and T. Nakanishi, 'Spectra in conformal field theories from the Rogers dilogarithm', Mod. Phys. Lett. A 7, 3487 (1992), (hep-th/9206034).

[34] F. Ravanini, R. Tateo and A. Valleriani, 'Dynkin TBAs', Int. J. Mod. Phys. A 8, 1707 (1993), hep-th/9207040.

[35] A. Kuniba, T. Nakanishi and J. Suzuki, 'Functional relations in solvable lattice models. 1: Functional relations and representation theory', Int. J. Mod. Phys. A 9, 5215 (1994), hep-th/9309137.

[36] A. Kuniba, T. Nakanishi and J. Suzuki, 'Functional relations in solvable lattice model 2: applications', Int. J. Mod. Phys. A 9, 5267 (1994), hep-th/9310060]. 
[37] A. Kuniba and J. Suzuki, 'Analytic Bethe Ansatz for fundamental representations of Yangians', Commun. Math. Phys. 173, 225 (1995), hep-th/9406180.

[38] S.L. Lukyanov, private communication (1999)

[39] P. Dorey, J. Suzuki and R. Tateo, 'Finite lattice Bethe ansatz systems and the Heun equation', J. Phys. A 37, 2047 (2004), hep-th/0308053.

[40] S.L. Lukyanov, 'Notes on parafermionic QFT's with boundary interaction', hep-th/0606155.

[41] K. Hornfeck, 'W algebras of negative rank', Phys. Lett. B 343, 94 (1995), hep-th/9410013.

[42] S.L. Lukyanov and V.A. Fateev, 'Exactly solvable models of conformal quantum theory associated with simple Lie algebra $\mathrm{D}(\mathrm{N})$ ', (In Russian) Sov. J. Nucl. Phys. 49, 925 (1989) [Yad. Fiz. 49, 1491 (1989)].

[43] P. Cvitanovic, 'Classics illustrated: Group Theory', Nordita notes; and Group Theory webbook at http://www.nbi.dk/GroupTheory/

[44] J. Suzuki, 'Spinons in magnetic chains of arbitrary spins at finite temperatures', J. Phys. A 32, 2341 (1999), cond-mat/9807076.

[45] C. Dunning, 'Finite size effects and the supersymmetric sine-Gordon models', J. Phys. A 36, 5463 (2003), hep-th/0210225.

[46] T.R. Klassen and E. Melzer, 'Purely elastic scattering theories and their ultraviolet limits', Nucl. Phys. B 338, 485 (1990).

[47] H.W. Braden, E. Corrigan, P. Dorey and R. Sasaki, 'Affine Toda field theory and exact S matrices', Nucl. Phys. B 338, 689 (1990).

[48] R. Tateo, 'The sine-Gordon model as $S O(n)_{1} \times S O(n)_{1} / S O(n)_{2}$ perturbed coset theory and generalizations', Int. J. Mod. Phys. A 10, 1357 (1995), hep-th/9405197.

[49] P. Dorey, R. Tateo and K.E. Thompson, 'Massive and massless phases in self-dual $Z_{N}$ spin models: some exact results from the thermodynamic Bethe ansatz', Nucl. Phys. B 470, 317 (1996), hep-th/9601123.

[50] L.N. Lipatov, 'Asymptotic behavior of multicolor QCD at high energies in connection with exactly solvable spin models', JETP Lett. 59, 596 (1994) [Pisma Zh. Eksp. Teor. Fiz. 59, 571 (1994)].

[51] J.A. Minahan and K. Zarembo, 'The Bethe-ansatz for $\mathrm{N}=4$ super Yang-Mills', JHEP 0303, 013 (2003), hep-th/0212208.

[52] G. Ferretti, R. Heise and K. Zarembo, 'New integrable structures in large-N QCD', Phys. Rev. D 70, 074024 (2004), hep-th/0404187]. 
[53] I.M. Gelfand and L.A. Dikii, 'Asymptotic behavior of the resolvent of Sturm-Liouville equations and the algebra of the Korteweg-De Vries equations', Russ. Math. Surveys 30, 77 (1975) [Usp. Mat. Nauk 30, 67 (1975)].

[54] J. Balog, L. Feher, L. O'Raifeartaigh, P. Forgacs and A. Wipf, 'Toda theory and W algebra from a gauged WZNW point of view', Annals Phys. 203, 76 (1990).

[55] P. Di Francesco, C. Itzykson and J.B. Zuber, 'Classical W algebras', Commun. Math. Phys. 140, 543 (1991).

[56] V.V. Bazhanov, S.L. Lukyanov and A.B. Zamolodchikov, 'Higher-level eigenvalues of Q-operators and Schrödinger equation', Adv. Theor. Math. Phys. 7, 711 (2004), hep-th/0307108.

[57] D. Fioravanti, 'Geometrical loci and CFTs via the Virasoro symmetry of the mKdV-SG hierarchy: an excursus', Phys. Lett. B 609, 173 (2005), [hep-th/0408079].

[58] Al.B. Zamolodchikov, 'Thermodynamic Bethe ansatz in relativistic models. Scaling three state Potts and Lee-Yang models', Nucl. Phys. B 342, 695 (1990).

[59] V.V. Bazhanov, S.L. Lukyanov and A.B. Zamolodchikov, 'Quantum field theories in finite volume: excited state energies', Nucl. Phys. B 489, 487 (1997), hep-th/9607099.

[60] P. Dorey and R. Tateo, 'Excited states by analytic continuation of TBA equations', Nucl. Phys. B 482, 639 (1996), hep-th/9607167).

[61] V. Gritsev, E. Altman, E. Demler, A. Polkovnikov, 'Full quantum distribution of contrast in interference experiments between interacting one dimensional Bose liquids', Nature Physics 2, 705 (2006), cond-mat/0602475.

[62] S.L. Lukyanov and P. Werner, 'Universal scaling behavior of the single electron box in the strong tunneling limit', cond-mat/0606453.

[63] V.V. Bazhanov, S.L. Lukyanov and A.M. Tsvelik, 'Analytical results for the Coqblin-Schrieffer model with generalized magnetic fields', Phys. Rev. B 68, 094427 (2003), cond-mat/0305237.

[64] A. Klümper, M.T. Batchelor and P.A. Pearce, 'Central charges of the 6and 19-vertex models with twisted boundary conditions', J. Phys. A 24, 3111 (1991).

[65] J. Heading, 'An introduction to phase-integral methods', (Methuen, 1962).

[66] J. Heading, 'Phase-integral methods I', Q. J1 Mech. Appl. Math. 15, 215 $(1962)$ 
_ 'Global phase-integral methods', Q. Jl Mech. Appl. Math. 30, 281 (1977).

[67] J. Heading, 'The Stokes Phenomenon II', Proc. Camb. Phil. Soc. 53, 419 (1957).

[68] P. Zinn-Justin, 'Non-linear integral equations for complex affine Toda models associated to simply laced Lie algebras', J. Phys. A 31, 6747 (1998), hep-th/9712222.

[69] P. Dorey, C. Dunning and R. Tateo, 'Spectral equivalences from Bethe ansatz equations', J. Phys. A 34, 5679 (2001), hep-th/0103051.

[70] A. Klümper and P.A. Pearce, 'Analytical calculations of scaling dimensions: tricritical Hard Square and critical Hard Hexagons', J. Stat. Phys. 64, 13 (1991).

[71] C. Destri and H.J. de Vega, 'New thermodynamic Bethe ansatz equations without strings', Phys. Rev. Lett. 69, 2313 (1992);

- 'Unified approach to thermodynamic Bethe ansatz and finite size corrections for lattice models and field theories', Nucl. Phys. B 438, 413 (1995), hep-th/9407117.

[72] J. Suzuki, 'Functional relations in Stokes multipliers - Fun with $x^{6}+\alpha x^{2}$ potential-', J. Statist. Phys. 102, 1029 (2001), quant-ph/0003066.

[73] C. Dunning, 'Massless flows between minimal W models', Phys. Lett. B 537, 297 (2002), hep-th/0204090. 